\title{
Upper bounds for the function solution of the homogeneous $2 D$ Boltzmann equation with hard potential
}

\author{
VLAD BALLY*
}

\begin{abstract}
We deal with $f_{t}(d v)$, the solution of the homogeneous $2 D$ Boltzmann equation without cutoff. The initial condition $f_{0}(d v)$ may be any probability distribution (except a Dirac mass). However, for sufficiently hard potentials, the semigroup has a regularization property (see [5]): $f_{t}(d v)=f_{t}(v) d v$ for every $t>0$. The aim of this paper is to give upper bounds for $f_{t}(v)$, the most significant one being of type $f_{t}(v) \leq C t^{-\eta} e^{-|v|^{\lambda}}$ for some $\eta, \lambda>0$.
\end{abstract}

Keywords: Boltzmann equation without cutoff, Hard potentials, Interpolation criterion, Integration by parts.

2010 MSC: 60H07, 60J75,82C40.

\section{Introduction and main results}

We are concerned with the solution of the two dimensional homogenous Boltzmann equation:

$$
\partial_{t} f_{t}(v)=\int_{\mathbb{R}^{2}} d v_{*} \int_{-\pi / 2}^{\pi / 2} d \theta\left|v-v_{*}\right|^{\gamma} b(\theta)\left(f_{t}\left(v^{\prime}\right) f_{t}\left(v_{*}^{\prime}\right)-f_{t}(v) f_{t}\left(v_{*}\right)\right) .
$$

Here $f_{t}(v)$ is a non-negative measure on $\mathbb{R}^{2}$ which represents the density of particles having velocity $v$ in a model for a gas in dimension two, and, with $R_{\theta}$ being the rotation of angle $\theta$,

$$
v^{\prime}=\frac{v+v_{*}}{2}+R_{\theta}\left(\frac{v-v_{*}}{2}\right), \quad v_{*}^{\prime}=\frac{v+v_{*}}{2}-R_{\theta}\left(\frac{v-v_{*}}{2}\right) .
$$

The function $b:\left[-\frac{\pi}{2}, \frac{\pi}{2}\right] \backslash\{0\} \rightarrow \mathbb{R}$ will be assumed to satisfy the following hypothesis:

$$
\begin{gathered}
\left(H_{\nu}\right) \quad \text { i) } \quad \exists 0<c<C \quad \text { s.t. } \quad c|\theta|^{-(1+\nu)} \leq b(\theta) \leq C|\theta|^{-(1+\nu)} \\
\text { ii) } \quad \forall k \in N, \exists C_{k} \quad \text { s.t. } \quad\left|b^{(k)}(\theta)\right| \leq C_{k}|\theta|^{-(k+1+\nu)} .
\end{gathered}
$$

In [17] it is proved that, for every $\nu \in\left(0, \frac{1}{2}\right)$ and $\gamma \in(0,1]$, the above equation has a unique weak solution. More precisely: under the assumption $\left(H_{\nu}\right)$ and the integrability condition $\int e^{|v|^{\lambda}} f_{0}(d v)<\infty$ for some $\lambda \in(\gamma, 2)$,[17] shows that there exists a unique weak solution $f_{t}$ of (1.1) which starts from $f_{0}$. Furthermore the solution satisfies $\sup _{t \leq T} \int e^{|v|^{\lambda^{\prime}}} f_{t}(d v)<\infty$ for every $\lambda^{\prime}<\lambda$. Throughout the paper these hypotheses are in force.

*Université Paris-Est, LAMA (UMR CNRS, UPEMLV, UPEC), INRIA, F-77454 Marne-la-Vallée, France. Email: bally@univ-mlv.fr 
Below, $f_{0}(d v)$ will be a probability distribution which is not assumed to be absolutely continuous with respect to the Lebesgue measure (we will just assume that $f_{0}(d v)$ is not a Dirac mass $\delta_{v_{0}}(d v)$ - in this trivial case the corresponding solution is $f_{t}(d v)=f_{0}(d v)=\delta_{v_{0}}(d v)$ for every $\left.t>0\right)$.

Our first aim is to give sufficient conditions under which, for every $t>0, f_{t}(d v)$ is absolutely continuous and to study the regularity of its density $f_{t}(v)$ : see Theorem 1.1 below. This problem has already been addressed in [5] for the same equation and under the same assumptions, in [16] for the three dimensional Boltzmann equation and in [1] for the Boltzmann equation in arbitrary dimension (however, in this last paper, $f_{0}(d v)$ is assumed to be absolutely continuous and to have finite entropy). In the case of Maxwell molecules (where $\gamma=0$ ) this problem is addressed in [20] and [13], and for Landau equation in [21]. These last three papers are the pioneering papers concerning the probabilistic approach to the regularity problem.

Our second aim is to give upper bounds for $f_{t}(v)$ : see Corollary 1.2 and Theorem 1.3 bellow. This is actually the main contribution of the paper. Two aspects of our bounds are noteworthy:

- for any $t>0, v \mapsto f_{t}(v)$ decays exponentially fast (spatial exponential decay) and

- $t \mapsto f_{t}(v)$ blows up at most polynomially as $t \rightarrow 0$ (blow-up in time).

Under a cutoff condition, and if the initial value is a function which is upper bounded by a Maxwellian potential, bounds on the spatial decay of $f_{t}$ were proved in [19]. Our result applies even when the initial condition is a measure (not necessarily absolutely continuos) and we work with the equation without cutoff. In [23] the author discusses upper bounds of polynomial type for an initial condition which is a smooth function. This is rather different from our framework, as our bounds are exponential and no regularity of the initial condition is required.

In order to precisely state our results, some notation is required. We denote by $\|\cdot\|_{p}$ respectively by $\|\cdot\|_{q, p}$ the norm in $L^{p}$ respectively in the Sobolev space $W^{q, p}$ on $\mathbb{R}^{2}$. For $p>1$ we denote by $p_{*}$ the conjugate of $p$. We fix $\nu \in\left(0, \frac{1}{2}\right)$ and $\gamma \in(0,1]$, we suppose that $f_{0}(d v)$ is not a Dirac mass, and that for some $\lambda \in(\gamma, 2)$ one has $\int e^{|v|^{\lambda}} f_{0}(d v)<\infty$.

We consider a non decreasing function $\rho: \mathbb{R}_{+} \rightarrow \mathbb{R}_{+}$such that $\rho(u)=1$ for $u \in(0,1), \rho(u)=u$ for $u \in(2, \infty)$ and $\rho \in C^{\infty}\left(\mathbb{R}_{+}\right)$and, for $0<\lambda^{\prime}<\lambda$, we define

$$
\Phi_{\lambda^{\prime}}(v)=e^{\rho\left(|v|^{\lambda^{\prime}}\right)} .
$$

The important point is that $\Phi_{\lambda^{\prime}}(v)=e^{|v|^{\lambda^{\prime}}}$ for $|v| \geq 2$; the function $\rho$ is used just to avoid singularities of the derivatives of $\Phi_{\lambda^{\prime}}$ around $v=0$. The specific choice of $\rho$ impacts just the constants $C$ (which anyway are not explicit).

Moreover we will use an auxiliary function $\varphi:[0, \infty) \rightarrow[0, \infty)$ defined by

$$
\varphi(\alpha)=\frac{(1-\nu)(1+\gamma+\alpha)}{1+\nu(\gamma+\alpha)}-1
$$

and we denote by $\alpha_{*}$ the unique solution of the equation $\varphi\left(\alpha_{*}\right)=\alpha_{*}$ (see (3.11) for the explicit value of $\left.\alpha_{*}\right)$. We also denote

$$
\eta=\frac{2(\varphi(2)-1)}{\varphi(2)-2}\left(\frac{13\left(1+\alpha_{*}\right)(2+\nu)}{\nu}-1\right) .
$$

We need to impose $\eta>0$. Direct computation shows that

$$
\begin{aligned}
& \varphi(0)>0 \quad \Leftrightarrow \quad \nu<\frac{\gamma}{2 \gamma+1} \\
& \varphi(2)>2 \quad \Leftrightarrow \quad \nu<\frac{\gamma}{4 \gamma+9}
\end{aligned}
$$


Theorem 1.1 A. Let us assume that $\nu<\frac{\gamma}{2 \gamma+1}$. Then the measure $f_{t}(d v)$ is absolutely continuous with respect to the Lebesgue measure on $\mathbb{R}^{2}$. We denote by $f_{t}(v)$ its density (that is $f_{t}(d v)=f_{t}(v) d v$ ). Let $\lambda^{\prime}<\lambda$.

$\boldsymbol{B}$ a. If $\nu<\frac{\gamma}{4 \gamma+9}$ then $\Phi_{\lambda^{\prime}} f_{t} \in L^{p}\left(\mathbb{R}^{2}\right)$ for every $p>1$ and (with $\eta$ given in (1.5))

$$
\left\|\Phi_{\lambda^{\prime}} f_{t}\right\|_{p} \leq \frac{C}{t^{\eta}}
$$

b. If $\frac{\gamma}{4 \gamma+9} \leq \nu<\frac{\gamma}{2 \gamma+1}$ then $\alpha_{*}<2$ and $\Phi_{\lambda^{\prime}} f_{t} \in L^{p}\left(\mathbb{R}^{2}\right)$ for every $1<p<\frac{2}{2-\alpha_{*}}$.

C.a If $\nu<\frac{\gamma}{4 \gamma+9}$ then $\Phi_{\lambda^{\prime}} f_{t} \in W^{q, p}\left(\mathbb{R}^{2}\right)$ for $q=1,2$ and $1<p<p_{q}$, with

$$
p_{1}=\frac{2(1+\nu(\gamma+2))}{1-\gamma+11 \nu+5 \nu \gamma} \quad \text { and } \quad p_{2}=\frac{2(1+\nu(\gamma+2))}{2-\gamma+13 \nu+6 \nu \gamma}
$$

Moreover for every $p<p_{q}$ one has

$$
\left\|\Phi_{\lambda^{\prime}} f_{t}\right\|_{q, p} \leq \frac{C}{t^{\eta}}
$$

b. If $\frac{\gamma}{4 \gamma+9} \leq \nu<\frac{\gamma}{3 \gamma+4}$ then $\Phi_{\lambda^{\prime}} f_{t} \in W^{1, p}\left(\mathbb{R}^{2}\right)$ for every $1<p<\frac{2}{3-\alpha_{*}}$.

We stress that the precise power $\eta$ in $t^{-\eta}$ in (1.8) and (1.10) is due to the technical approach that we use. We do not expect it to be optimal (see the point $D$ in Lemma 3.1 for more precise estimates, which themselves are not optimal). However this guarantees that the blow up of $f_{t}$ as $t \rightarrow 0$ is at most polynomial.

In order to be able to compare this result with the ones in the papers which we quoted before, take $s>1$ and $\nu=\frac{2}{s-1}, \gamma=\frac{s-5}{s-1}$ : these are the values which are significant in the case of the three dimensional Boltzmann equation. Our condition $\gamma>0$ implies that $s>5$; in the literature this case is known as the "hard potential" case. With this choice of $\nu$ and of $\gamma$ we have $\nu<\frac{\gamma}{2 \gamma+1}$ iff $s>9$ and $\nu<\frac{\gamma}{4 \gamma+9}$ iff $s>16+\sqrt{193} \sim 30$. The regularity results of the above theorem are analogous with the ones in [5], though not identical. In [16] one deals with the real three dimensional equation (without cutoff) and obtains absolute continuity for a larger range for $s$ then in the above theorem. However the $L^{p}$ estimates obtained in our paper are stronger: we obtain $\Phi_{\lambda^{\prime}} f_{t} \in L^{p}\left(\mathbb{R}^{2}\right)$ instead of $f_{t} \in L^{2}\left(\mathbb{R}^{2}\right)$. Moreover, we obtain bounds depending polynomially on $t \downarrow 0$. The result of $[1]$ is stronger in the sense that it applies to equations in any dimension, but it requires that the initial condition is already a function (so it is not really possible to compare them).

We give now some consequences of the previous result concerning the tails of $f_{t}(d v)$ :

Corollary 1.2 Suppose that $\nu<\frac{\gamma}{2 \gamma+1}$. For every $\lambda^{\prime}<\lambda$ there exists a constant $C \geq 1$ (depending on $\left.\lambda^{\prime}\right)$ such that for every $R>1, t \in(0,1]$

$$
\begin{gathered}
f_{t}(\{v:|v| \geq R\}) \leq \frac{C}{t^{\kappa}} e^{-R^{\lambda^{\prime}}} \quad \text { with } \\
\quad \kappa=\frac{13(2+\nu)(1-\nu)(1+\gamma)}{\nu(1+\nu \gamma)}-1 .
\end{gathered}
$$

We give now the upper bound for $f_{t}(v)$ :

Theorem 1.3 Suppose that $\nu<\frac{\gamma}{4 \gamma+9}$. Then $p_{1}>2$ (given in (1.9)) and $f_{t} \in C^{0, \chi}$ (Hölder continuous functions of order $\chi$ ) with $\chi=1-\frac{2}{p_{1}}$ for all $t>0$. Moreover for every $\lambda^{\prime}<\lambda$ there exists $C \geq 1$ such that

$$
\left|f_{t}(v)\right| \leq \frac{C}{t^{\eta}} e^{-|v|^{\lambda^{\prime}}}
$$

with $\eta$ given in (1.5). Finally, there exists $C \geq 1$ such that for every $v, w \in \mathbb{R}^{2}$ with $|w-v| \leq 1$

$$
\left|f_{t}(w)-f_{t}(v)\right| \leq \frac{C}{t^{\eta}} e^{-|v|^{\lambda^{\prime}}}|w-v|^{\chi} .
$$


To our best knowledge, both the time-space estimate (1.13) and the Hölder continuity of $f_{t}$ and the estimate (1.14) are new. For the Landau equation, lower and upper bounds for the solution have been obtained in [21] using integration by parts techniques based on the classical Malliavin calculus. This approach is not directly possible in our framework because of the singularities that appear in the problem. We will use similar but ultimately different techniques below.

Corollary 1.2 and Theorem 1.3 are the main contributions of our paper. The drawback of our approach is that it applies only to "very hard potentials" $(s>9$ for (1.11) respectively $s>30$ for (1.13) and (1.14)). Moreover, the exponent $\eta$ in the polynomial blow-up $t^{-\eta}$ is not expected to be optimal.

The proofs are based on a "balance argument" which is interesting in itself, and may be useful in other settings. We summarize it below.

Consider a family of random variables $F_{\varepsilon} \sim f_{\varepsilon}(v) d v, \varepsilon>0$ and a random variable $F$. Suppose that $F_{\varepsilon}-F \rightarrow 0$ and $f_{\varepsilon} \uparrow \infty$ as $\varepsilon \rightarrow 0$, in a certain sense. If the convergence to zero is sufficiently faster then the blow-up of $f_{\varepsilon}$, then one is able to prove that the law of $F$ has a density and to obtain some regularity of the density. This idea first appears in [18] and has been used ever since in several papers (see [12] for example). In these papers the "balance" between the speed of convergence to zero and the blow-up is built by using Fourier analysis. Later on, in [11] the authors introduced a new method based on a Besov space criterion, which turns out to be significantly more powerful then the one based on Fourier analysis. This is the method used in [16] in the case of the three dimensional Boltzmann equation (see also [10]). Finally, in [2], a third method which is close to interpolation theory was introduced.

The criterion that we use in the present paper is an improvement of the latter method: we give an abstract framework in which an integration by parts formula can be applied and we quantify the blow-up of $f_{\varepsilon}$ in terms of the weights appearing in the corresponding integration by parts formula. Let us be more precise.

Consider a family of random variables $F_{\varepsilon}$ with values in $\mathbb{R}^{d}$ and $G_{\varepsilon}$ with values in $[0,1], \varepsilon>0$. Associate to them the measures $\mu_{\varepsilon}$ given by

$$
\int \varphi d \mu_{\varepsilon}=E\left(\varphi\left(F_{\varepsilon}\right) G_{\varepsilon}\right)
$$

The random variables $G_{\varepsilon}$ play a technical role, and will be used in some localization procedure.

We assume that for every $\varepsilon>0$ and every multi-index $\alpha$ one may find a random variable $H_{\alpha, \varepsilon}$ such that the following integration by parts formula holds:

$$
E\left(\partial^{\alpha} \varphi\left(F_{\varepsilon}\right) G_{\varepsilon}\right)=E\left(\varphi\left(F_{\varepsilon}\right) H_{\alpha, \varepsilon}\right) \quad \forall \varphi \in C_{b}^{\infty}\left(\mathbb{R}^{d}\right) .
$$

Here $\alpha=\left(\alpha_{1}, \ldots, \alpha_{m}\right) \in\{1, \ldots, d\}^{m}$, is a multi-index of length $|\alpha|=m$ and $\partial^{\alpha}$ is the derivative associated to $\alpha$.

Additionally, we assume that $H_{\alpha, \varepsilon}$ may be chosen such that, for every $q \in \mathbb{N}$ and $p>1$, there exist some constants $\widehat{H}_{q, p}$ and $a, b, \varepsilon_{*} \geq 0$ such that for every $0<\varepsilon<\varepsilon_{*}$

$$
\sup _{|\alpha| \leq q}\left\|H_{\alpha, \varepsilon}\right\|_{p} \leq \widehat{H}_{q, p} \varepsilon^{-b(q+a)}
$$

In particular this implies that $\mu_{\varepsilon}(d v)=f_{\varepsilon}(v) d v$ with $f_{\varepsilon} \in C^{\infty}\left(\mathbb{R}^{d}\right)$.

Moreover, we consider a random variable $F \in R^{d}$ and we assume that there exists $\beta>0$ and $C_{*} \geq 1$ such that

$$
\left\|G_{\varepsilon}-1\right\|_{2}+\left\|F_{\varepsilon}-F\right\|_{1} \leq C_{*} \varepsilon^{\beta} .
$$

Finally we consider a function $\Phi: \mathbb{R}^{d} \rightarrow \mathbb{R}_{+}$which belongs to $C^{\infty}\left(\mathbb{R}^{d}\right)$, is convex and there exists $C \geq 1$ such that $\Phi(x) \geq \frac{1}{C} \Phi(y)$ if $|x| \geq|y|$. Moreover we assume that for each $h \in \mathbb{N}$ and for each multi-index $\alpha$ there exist some constants $c_{1}, c_{2}$ (depending on $h$ and $\alpha$ ) such that

$$
(1+|x|)^{h}\left(1+\left|\partial^{\alpha} \Phi(x)\right|\right) \leq c_{1} \Phi^{c_{2}}(x) .
$$


The typical examples are $\Phi(x)=\left(1+|x|^{2}\right)^{r}$ and $\Phi_{\lambda}$ defined in (1.3). We denote by $\mathcal{C}$ the class of these functions and for $\Phi \in \mathcal{C}$ and $\theta \geq 0$ we denote

$$
C_{\theta}(\Phi)=E\left(\Phi^{\theta}(F)\right)+\sup _{\varepsilon>0} E\left(\Phi^{\theta}\left(F_{\varepsilon}\right)\right) .
$$

Our criteria are the following:

Theorem 1.4 A. Let $F \in R^{d}$ be a random variable. Suppose that one is able to find a family $F_{\varepsilon} \in \mathbb{R}^{d}$ and $G_{\varepsilon} \in \mathbb{R}, \varepsilon>0$ which verify (1.15), (1.16) and (1.17). Fix $q \in \mathbb{N}$ and $p>1$ and assume that (recall that $p_{*}$ is the conjugate of $p$ )

$$
\beta>b\left(1+q+\frac{d}{p_{*}}\right)
$$

Then $P(F \in d x)=f(x) d x$ with $f \in W^{q, p}\left(\mathbb{R}^{d}\right)$.

B. Consider a function $\Phi \in \mathcal{C}$ such that $C_{\theta}(\Phi)<\infty$ for every $\theta>0$. Let $q \in \mathbb{N}$ and $p>1, \delta>0$ be given. Assume that (1.20) holds. There exist some constants $C \geq 1, \theta \geq 1$ and $h_{*} \geq 1$ (depending on $q, d, \beta, b, p$ and $\delta$ ) such that for $h \geq h_{*}$ one has

$$
\begin{aligned}
\|\Phi f\|_{q, p} & \leq \Gamma_{\Phi, \theta}(q, h, p) \text { with } \\
\Gamma_{\Phi, \theta}(q, h, p) & :=C \times\left(C_{*}+C_{\theta}(\Phi)\right) \times\left(h^{2 b} \widehat{H}_{2 h+q+d, p_{*}}^{1 / 2 h}\right)^{(1+\delta)\left(1+q+d / p_{*}\right)}
\end{aligned}
$$

with $C_{*}$ given in (1.17), $\widehat{H}_{2 h+q+d, p_{*}}$ given in (1.16) and $C_{\theta}(\Phi)$ given in (1.19).

C. Suppose that (1.20) holds for $q=1$ and $p>d$. Then $f \in C^{0, \chi}\left(\mathbb{R}^{d}\right)$ with $\chi=1-\frac{d}{p}$ and we have

$$
|f(x)| \leq \frac{1}{\Phi(x)} \times\|\Phi f\|_{1, p} \leq \frac{1}{\Phi(x)} \times \Gamma_{\Phi, \theta}(1, h, p) .
$$

Moreover, let

$$
\widehat{\nabla} \Phi(x)=\sup _{|x-y| \leq 1}|\nabla \Phi(y)| .
$$

For every $x, y \in \mathbb{R}^{d}$ with $|x-y| \leq 1$

$$
\begin{aligned}
|f(y)-f(x)| & \leq \frac{1}{\Phi(x)}\left(1+\frac{\widehat{\nabla} \Phi(x)}{\Phi(x)}\right) \times\|\Phi f\|_{1, p} \times|x-y|^{\chi} \\
& \leq \frac{1}{\Phi(x)}\left(1+\frac{\widehat{\nabla} \Phi(x)}{\Phi(x)}\right) \times \Gamma_{\Phi, \theta}(1, h, p) \times|x-y|^{\chi}
\end{aligned}
$$

Remark 1.5 The constants in the previous theorem depend on $\Phi$ by means of the constants $c_{1}$ and $c_{2}$ which appear in the property (1.18).

Remark 1.6 The estimates in the point $\boldsymbol{C}$ in the above theorem are quite precise and this is important in order to prove Theorem 1.3. But, roughly speaking, (1.23) reads

$$
|f(x)| \leq \frac{C}{\Phi(x)} \times\left(E\left(\Phi^{\theta}(F)\right)+\sup _{\varepsilon>0} E\left(\Phi^{\theta}\left(F_{\varepsilon}\right)\right)\right) .
$$

The constant $C$ depends on $h_{*}, \widehat{H}_{2 h_{*}+1+d, p_{*}}^{1 / 2 h}, \beta, C_{*}, d$ and $p$. This version is less precise but focus on the following basic fact: if one is able to estimate the moments $E\left(\Phi^{\theta}(F)\right)$ and $E\left(\Phi^{\theta}\left(F_{\varepsilon}\right)\right)$ then one obtains the upper bound of $f$ by $\Phi^{-1}(x)$. This means that one is able to translate moment estimates in terms of upper bounds for the density function. 
Let us try to give the heuristic which is behind the above criterion. Suppose for simplicity that we are in dimension $d=1$ and that $F$ itself satisfies the integration by parts formula $(1.15)$ (with $G=1$ ). We formally write

$$
f_{F}(x)=E\left(\delta_{0}(F-x)\right)=E\left(1_{[0, \infty)}^{\prime}(F-x)\right)=E\left(1_{[0, \infty)}(F-x) H_{1}\right) .
$$

Using regularization by convolution the above reasoning may be done rigorously and it proves that $P(F \in d x)=f_{F}(x) d x$. Let us now compute the upper bounds. Take for example $\Phi(x)=e^{|x|}$. Then, using Schwarz's inequality first and Chebishev's inequality then

$$
f_{F}(x) \leq P^{1 / 2}(F \geq x)\left\|H_{1}\right\|_{2}=P^{1 / 2}\left(e^{|F|} \geq e^{|x|}\right)\left\|H_{1}\right\|_{2} \leq e^{-\frac{1}{2}|x|} E\left(e^{|F|}\right)^{1 / 2}\left\|H_{1}\right\|_{2}
$$

so we obtain an estimate of type (1.23).

The classical probabilistic way to obtain integration by parts formulas of type (1.15) is to use Malliavin calculus - our approach is strongly inspired from this methodology, but however, at a certain point, takes a completely different direction. Malliavin calculus is an infinite differential calculus settled in the following way. One considers a class of "simple functionals" which are "finite dimensional" objects. For them one defines a derivative operator $D$ and a divergence operator $L$ using the classical differential operators in finite dimension. Then one defines the extension of these operators in infinite dimension: a general functional $F$ is in the domain of $D$ (respectively of $L$ ) if one may find a sequence of simple functionals $F_{\varepsilon}$ such that $F_{\varepsilon} \rightarrow F$ in $L^{2}$ and $D F_{\varepsilon} \rightarrow U$ in $L^{2}$ (respectively $L F_{\varepsilon} \rightarrow V$ ). Then one defines $D F=\lim _{\varepsilon \rightarrow 0} D F_{\varepsilon}$ respectively $L F=\lim _{\varepsilon \rightarrow 0} L F_{\varepsilon}$. These operators are used in order to built the weight $H_{1}$ in the integration by parts formula $E\left(\varphi^{\prime}(F)\right)=E\left(\varphi(F) H_{1}\right)$. In our approach we also settle a finite dimensional calculus as above so we define $D F_{\varepsilon}$ and $L F_{\varepsilon}$ for a finite dimensional $F_{\varepsilon}$ and we use them in order to obtain $E\left(\varphi^{\prime}\left(F_{\varepsilon}\right)\right)=E\left(\varphi\left(F_{\varepsilon}\right) H_{1, \varepsilon}\right)$ (see Section 5). But in our framework $L F_{\varepsilon} \uparrow \infty$ (see Remark 5.1 for more details) so $F$ is no more in the domain of $L$. So the second step in Malliavin's methodology brakes down: there is no infinite dimensional calculus available here. And we have $H_{1, \varepsilon} \uparrow \infty$. But, if we are able to obtain the estimates given in (1.16) and in (1.17) and if the equilibrium condition (1.20) is verified, then we still obtain the regularity of the law of $F$ and the upper bound for its density. This is the object of Theorem 1.4. The ideas of this criterion origin in [2] and the proof is given in Section 4.

In the years $80^{\prime}$ th starting with the papers [8], [24] and [7] a version of Malliavin calculus for Poisson point measures has been developed and successfully used in order to study the regularity of the solutions of $S D E^{\prime} s$ with jumps (see also [22] and [9] and the references there for recent developments in this area). In the above papers the extension form the finite dimensional calculus to the infinite

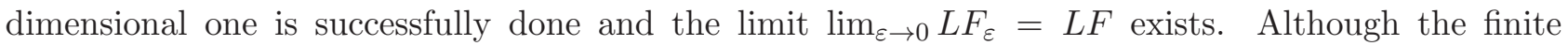
dimensional calculus developed in our paper is similar, in our framework $L F_{\varepsilon}$ blows up as $\varepsilon \rightarrow 0$. This is because the law of the jumps in Boltzmann equation (and more generally in the framework of Piecewise Deterministic Markov Processes) depends on the position of the particle before the jump while for usual $S D E^{\prime} s$, the law of the jumps is independent of the position of the particle (see Section 5 for details).

The proof of Theorem 1.1 is based on the criterion given in Theorem 1.4. In order to do it, following Tanaka [26], we introduce a stochastic equation which represents the probabilistic representation of the Boltzmann equation and we construct some regularized version of this equation. The solutions of these equations play the role of $F$ and of $F_{\varepsilon}$ in Theorem 1.4. Then we recall two results from [5]: the first one permits to estimate the error in (1.17) and the second one gives the integration by parts formula (1.15) and the estimates in (1.16).

The paper is organized as follows: in Section 2 we recall the results from [5] and in Section 3 we prove Theorem 1.1, Corollary 1.2 and Theorem 1.3 (starting from the general criterion given in Theorem 1.4). In Section 4 we prove Theorem 1.4. In Section 5 we give an overview of the results from [5] and we precise the changes which are necessary in order to obtain an explicit expression for the dependence with respect to $t$ of the constants in the main estimates. 


\section{Preliminary results}

In this section we present some results from [5]. Throughout this section we fix $\nu \in\left[0, \frac{1}{2}\right), \gamma \in[0,1]$ and $\lambda \in(\gamma, 2)$ and the corresponding solution $f_{t}(d v)$ of (1.1) (which exists and is unique). In [5] (following the ideas from [26]) one gives the probabilistic interpretation of the equation (1.1). We recall this now. Let $E=\left[-\frac{\pi}{2}, \frac{\pi}{2}\right] \times \mathbb{R}^{2}$ and let $N(d t, d \theta, d v, d u)$ be a Poisson point measure on $E \times \mathbb{R}_{+}$ with intensity measure $d t \times b(\theta) d \theta \times f_{t}(d v) \times d u$. Consider also the matrix

$$
A(\theta)=\frac{1}{2}\left(\begin{array}{ll}
\cos \theta-1 & -\sin \theta \\
\sin \theta & \cos \theta-1
\end{array}\right)=\frac{1}{2}\left(R_{\theta}-I\right) .
$$

We are interested in the equation

$$
V_{t}=V_{0}+\int_{0}^{t} \int_{E \times \mathbb{R}_{+}} A(\theta)\left(V_{s-}-v\right) 1_{\left\{u \leq\left|V_{s-}-v\right|^{\gamma}\right\}} N(d s, d \theta, d v, d u)
$$

with $P\left(V_{0} \in d v\right)=f_{0}(d v)$. Proposition 2.1 in [5] asserts that the equation (2.1) has a unique càdlàg solution $\left(V_{t}\right)_{t \geq 0}$ and $P\left(V_{t} \in d v\right)=f_{t}(d v)$ (in this sense $V_{t}$ represents the probabilistic representation for $f_{t}$ ).

In order to handle the equation (2.1) we face several difficulties: the derivatives of the function $w \rightarrow|w-v|^{\gamma}$ blow up in the neighborhood of $v$ - so we have to use a regularization procedure. Moreover, this function is unbounded and so we use a truncation argument. Finally, the measure $\theta^{-(1+\nu)} d \theta$ has infinite mass, and it is convenient to use a truncation argument also. We follow here the ideas and results from [5]. We fix

$$
\eta_{0} \in\left(\frac{1}{\lambda}, \frac{1}{\gamma \vee \nu}\right) \quad \text { and } \quad \Gamma_{\varepsilon}=\left(\ln \frac{1}{\varepsilon}\right)^{\eta_{0}} .
$$

Since $\gamma \eta_{0}>1$ we have, for every $C \geq 1$ and $a>0$

$$
\varlimsup_{\varepsilon \rightarrow 0} \varepsilon^{a} e^{C \Gamma_{\varepsilon}^{\gamma}}=0
$$

So $e^{C \Gamma_{\varepsilon}^{\gamma}} \leq \varepsilon^{-a}$ for sufficiently small $\varepsilon$. Moreover, if $\kappa>0$ is such that $\kappa \eta_{0}>1$, then for every $A \geq 1$

$$
\varlimsup_{\varepsilon \rightarrow 0} \varepsilon^{-A} e^{-\Gamma_{\varepsilon}^{\kappa}}=0 .
$$

So $e^{-\Gamma_{\varepsilon}^{\kappa}} \leq \varepsilon^{A}$ for sufficiently small $\varepsilon$.

We construct the following approximation. We consider a $C^{\infty}$ even non negative function $\chi$ supported by $[-1,1]$ and such that $\int_{R} \chi(x) d x=1$ and we define

$$
\varphi_{\varepsilon}(x)=\int_{R}\left((y \vee 2 \varepsilon) \wedge \Gamma_{\varepsilon}\right) \frac{\chi((x-y) / \varepsilon)}{\varepsilon} d y .
$$

Observe that we have $2 \varepsilon \leq \varphi_{\varepsilon}(x) \leq \Gamma_{\varepsilon}$ for every $x \in \mathbb{R}, \varphi_{\varepsilon}(x)=x$ for $x \in\left(3 \varepsilon, \Gamma_{\varepsilon}-1\right), \varphi_{\varepsilon}(x)=2 \varepsilon$ for $x \in(0, \varepsilon)$ and $\varphi_{\varepsilon}(x)=\Gamma_{\varepsilon}$ for $x \in\left(\Gamma_{\varepsilon}, \infty\right)$. To the cut off function $\varphi_{\varepsilon}$ one associates the equation

$$
V_{t}^{\varepsilon}=V_{0}+\int_{0}^{t} \int_{E \times \mathbb{R}_{+}} A(\theta)\left(V_{s-}^{\varepsilon}-v\right) 1_{\left\{u \leq \varphi_{\varepsilon}^{\gamma}\left(\left|V_{s-}^{\varepsilon}-v\right|\right)\right\}} N(d s, d \theta, d v, d u) .
$$

We construct a second approximation: for $\zeta>0$ we consider a smooth cut-off function $I_{\zeta}$ which is a smooth version of $1_{\{|\theta|>\zeta\}}$ (the precise definition is given in (5.2)) and we associate the equation

$$
V_{t}^{\varepsilon, \zeta}=V_{0}+\int_{0}^{t} \int_{E \times \mathbb{R}_{+}} A(\theta)\left(V_{s-}^{\varepsilon, \zeta}-v\right) 1_{\left\{u \leq \varphi_{\varepsilon}^{\gamma}\left(\left|V_{s-}^{\varepsilon, \zeta}-v\right|\right)\right\}} I_{\zeta}(\theta) N(d s, d \theta, d v, d u) .
$$


We state now a property which will be used in the following: given $\alpha \in[0,2]$ and $\kappa \geq 0$ there exists $K \geq 1$ such that for every $w \in \mathbb{R}^{2}, t_{0}>0$ and every $0<\varepsilon<1$

$$
\left(A_{\alpha, \kappa}\right) \quad \sup _{t_{0} \leq t \leq T} f_{t}(\operatorname{Ball}(w, \varepsilon)) \leq \frac{K}{t_{0}^{\kappa}} \varepsilon^{\alpha} .
$$

Since $f_{t}(d v)$ is a probability measure, this property is always verified with $K=1, \alpha=0$ and $\kappa=0$. In Proposition 2.1 from [5] one proves that the equations (2.6) and (2.7) have a unique solution and

$$
E\left|V_{t}^{\varepsilon, \zeta}-V_{t}^{\varepsilon}\right| \leq C_{T} e^{C \Gamma_{\varepsilon}^{\gamma}} \times \zeta^{1-\nu} \times t \quad \forall t \leq T .
$$

Moreover, if $\left(A_{\alpha, \kappa}\right)$ holds, then

$$
E\left|V_{t}-V_{t}^{\varepsilon}\right| \leq C_{T} e^{C \Gamma_{\varepsilon}^{\gamma}} \times \varepsilon^{1+\gamma+\alpha} \times t^{1-\kappa} \quad \forall t \leq T .
$$

We stress that in [5] the explicit dependence on the time $t$ does not appear in the right hand side of the above estimates - but a quick glance to the proof shows that we have the dependence on $t$ as in (2.9) and in (2.10) ( this is important if we look to short time behavior). Moreover, in the same proposition one proves that for every $0<\lambda^{\prime}<\lambda$ there exists some $\varepsilon_{0}>0$ such that

$$
\sup _{\varepsilon \leq \varepsilon_{0}} \sup _{\zeta \leq 1} E\left(\sup _{t \leq T}\left(e^{\left|V_{t}\right|^{\lambda^{\prime}}}+e^{\left|V_{t}^{\varepsilon}\right|^{\lambda^{\prime}}}+e^{\left|V_{t}^{\zeta, \varepsilon}\right|^{\lambda^{\prime}}}\right)=: C\left(\lambda^{\prime}\right)<\infty .\right.
$$

Finally in Theorem 4.1 in [5] one proves an integration by parts formula that we present now. One defines (see (4.1) and (4.2) in [5]) a random process $G_{t}^{\varepsilon, \zeta}$ which verifies

$$
1_{\left\{\sup _{s \leq t}\left|V_{s}^{\zeta, \varepsilon}\right| \leq \Gamma_{\varepsilon}-1\right\}} \leq G_{t}^{\varepsilon, \zeta} \leq 1_{\left\{\sup _{s \leq t}\left|V_{s}^{\zeta, \varepsilon}\right| \leq \Gamma_{\varepsilon}\right\}}
$$

The precise form of $G_{t}^{\varepsilon, \zeta}$ is not important here - the only property which we need is (2.12). Moreover, since the law of $V_{t}^{\varepsilon, \zeta}$ is not absolutely continuous we use the following regularization procedure. One considers a two dimensional standard normal random variable $Z$ and denotes

$$
F_{t}^{\varepsilon, \zeta}=\sqrt{u_{\zeta}(t)} Z+V_{t}^{\varepsilon, \zeta} \text { with } \quad u_{\zeta}(t)=t \zeta^{4+\nu} .
$$

Then one proves (see (4.3) and (4.4) in [5]) that for every multi-index $\beta \in\{1,2\}^{q}$ there exists a random variable $K_{\beta}\left(F_{t}^{\varepsilon, \zeta}, G_{t}^{\varepsilon, \zeta}\right)$ such that for every function $\psi \in C^{q}\left(\mathbb{R}^{2}\right)$

$$
E\left(\partial^{\beta} \psi\left(F_{t}^{\varepsilon, \zeta}\right) G_{t}^{\varepsilon, \zeta}\right)=E\left(\psi\left(F_{t}^{\varepsilon, \zeta}\right) K_{\beta}\left(F_{t}^{\varepsilon, \zeta}, G_{t}^{\varepsilon, \zeta}\right)\right) .
$$

One also proves that for every $q \in N$ and every $\kappa \in\left(\frac{1}{\eta_{0}}, \lambda\right)$ one may find a constant $C$ (depending on $q$ and $\kappa$ only) such that for every $p \geq 1$

$$
\left\|K_{\beta}\left(F_{t}^{\varepsilon, \zeta}, G_{t}^{\varepsilon, \zeta}\right)\right\|_{p} \leq \frac{C}{t^{\frac{2+\nu}{\nu}(12 q-4)}} e^{C \Gamma_{\varepsilon}^{\gamma}}\left(\varepsilon^{-q} \zeta^{-\nu q}+e^{-\Gamma_{\varepsilon}^{\kappa}} \zeta^{-2 \nu q}\right)
$$

In particular this gives for every function $\psi \in C^{q}\left(\mathbb{R}^{2}\right)$ and every multi-index $\beta \in\{1,2\}^{q}$

$$
\left|E\left(\partial^{\beta} \psi\left(F_{t}^{\varepsilon, \zeta}\right) G_{t}^{\varepsilon, \zeta}\right)\right| \leq \frac{C}{t^{\frac{2+\nu}{\nu}(12 q-4)}} e^{C \Gamma_{\varepsilon}^{\gamma}}\left(\varepsilon^{-q} \zeta^{-\nu q}+e^{-\Gamma_{\varepsilon}^{\kappa}} \zeta^{-2 \nu q}\right) \times\|\psi\|_{\infty} .
$$

The proof of (2.14) and (2.15) is based on a Malliavin type calculus for jump processes and is quit technical. In Section 5 we give an overview of the objects which come on in this proof and on the main estimates which are needed. In particular we mention that in Theorem 4.1 in [5] the dependence with respect to $t$ in the right hand side of (2.15) is not explicit - at the end of Section 5 we precise this dependence (see (5.15)). 


\section{Proof of Theorem 1.1}

In the following we adapt the results presented in the previous section to our specific goals. Recall that the parameters $\nu \in\left[0, \frac{1}{2}\right), \gamma \in[0,1]$ and $\lambda \in(\gamma, 2)$ are given and characterizes the unique solution $f_{t}(d v)$ of (1.1). Suppose that $\left(A_{\alpha, \kappa}\right)$ (see (2.8)) holds for some $\alpha \geq 0$ and $\kappa \geq 0$. In order to equilibrate the errors in (2.9) and (2.10) we take

$$
\zeta=\zeta_{\alpha}(\varepsilon)=\varepsilon^{(1+\gamma+\alpha) /(1-\nu)} .
$$

With this choice, for every $c>0$, we may find $C \geq 1$ (depending on $c$, see $((2.3))$ such that

$$
E\left(\left|V_{t}-V_{t}^{\varepsilon, \zeta_{\alpha}(\varepsilon)}\right|\right) \leq \frac{C}{t^{\kappa-1}} \times e^{C \Gamma_{\varepsilon}^{\gamma}} \times \varepsilon^{1+\gamma+\alpha} \leq \frac{C}{t^{\kappa-1}} \varepsilon^{1+\gamma+\alpha-c} .
$$

Recall that $\eta_{0}$ is given in (2.2) and $\lambda \eta_{0}>1$. So we may choose (and fix) some $\lambda^{\prime} \in\left(\frac{1}{\eta_{0}}, \lambda\right)$. We work with the function $\Phi_{\lambda^{\prime}}$ given in (1.3) and we define

$$
g_{t}(d v)=\Phi_{\lambda^{\prime}}(v) f_{t}(d v) .
$$

Moreover, for $\varepsilon>0$, we recall that $F_{t}^{\varepsilon, \zeta}$ and $G_{t}^{\varepsilon, \zeta}$ are given in (2.13) and (2.12), and we define $f_{t}^{\varepsilon, \alpha}(d v)$ and $g_{t}^{\varepsilon, \alpha}(d v)$ by

$$
\int \psi(v) f_{t}^{\varepsilon, \alpha}(d v)=E\left(\psi\left(F_{t}^{\varepsilon, \zeta_{\alpha}(\varepsilon)}\right) G_{t}^{\varepsilon, \zeta_{\alpha}(\varepsilon)}\right), \quad g_{t}^{\varepsilon, \alpha}(d v)=\Phi_{\lambda^{\prime}}(v) f_{t}^{\varepsilon, \alpha}(d v) .
$$

In (1.4) we introduced the function $\varphi$. Notice that $\varphi$ solves the equation

$$
1+\gamma+\alpha-(1+\varphi(\alpha)) \frac{1+\nu(\gamma+\alpha)}{1-\nu}=0 .
$$

We construct the sequences

$$
\alpha_{k+1}=\varphi\left(\alpha_{k}\right), \quad \kappa_{k+1}=\kappa_{k}-1+\frac{13(2+\nu)}{\nu}\left(1+\alpha_{k+1}\right)
$$

with $\alpha_{0}=0$ and $\kappa_{0}=0$. Direct computation shows that $\varphi^{\prime}(\alpha)>0$ for every $\alpha$, so $\varphi$ is strictly increasing. We will assume in the following that $\alpha_{1}=\varphi(0)>0=\alpha_{0}$ and this implies $\alpha_{k+1}>\alpha_{k}$ for every $k$. It follows that $\alpha_{k} \uparrow \alpha_{*}$ solution of $\varphi\left(\alpha_{*}\right)=\alpha_{*}$ (see (3.11) for the explicit value of $\alpha_{*}$ ). Notice also that $\alpha_{1}=\varphi(0)>0$ is equivalent with $\nu<\frac{\gamma}{2 \gamma+1}$ and $\varphi(2)>2$ is equivalent with $\nu<\frac{\gamma}{4 \gamma+9}$.

We know that $\left(A_{0,0}\right)$ holds. Our aim now is to employ Theorem 1.4 in order to obtain $\left(A_{\alpha, \kappa}\right)$ for $\alpha$ as large as possible.

Lemma 3.1 A. Let $q \in \mathbb{N}, \alpha \in[0,2]$ and $\kappa \geq 0$ be given. Suppose that $\left(A_{\alpha, \kappa}\right)$ holds with $\varphi(\alpha)>q$, and take $p>1$ such that

$$
q+\frac{2}{p_{*}}<\varphi(\alpha)
$$

Then $f_{t}(d v)=f_{t}(v) d v$ with $f_{t} \in W^{q, p}$. Moreover, for every $\lambda^{\prime}<\lambda$ there exists $C \geq 1$ such that

$$
\left\|\Phi_{\lambda^{\prime}} f_{t}\right\|_{q, p} \leq \frac{C}{t^{\kappa-1+\frac{13(2+\nu)}{\nu}(1+\varphi(\alpha))}}
$$

$\boldsymbol{B}$ Suppose that $\left(A_{\alpha, \kappa}\right)$ holds and $\varphi(\alpha)>0$. Then $\left(A_{\alpha^{\prime}, \kappa^{\prime}}\right)$ holds for every $\alpha^{\prime}<\varphi(\alpha) \wedge 2$ with

$$
\kappa^{\prime}=\kappa-1+\frac{13(2+\nu)}{\nu}(1+\varphi(\alpha)) .
$$


C. Let $\alpha_{k}, \kappa_{k}, k \in \mathbb{N}$ be the sequences defined in (3.3). Suppose that $\varphi(0)>0$. Then, for each $k \in \mathbb{N}_{*}$ the property $\left(A_{\alpha, \kappa_{k}}\right)$ holds for every $\alpha<\alpha_{k} \wedge 2$.

D. Suppose that $\varphi(0)>0$. Let $k, q \in \mathbb{N}$ and $p>1$ be such that

$$
q+\frac{2}{p_{*}}<\varphi\left(\alpha_{k} \wedge 2\right)=\alpha_{k+1} \wedge \varphi(2) .
$$

Then

$$
\left\|\Phi_{\lambda^{\prime}} f_{t}\right\|_{q, p} \leq \frac{C}{t^{\kappa_{k+1}}}
$$

Proof of A. We will use Theorem 1.4 with $d=2$, and $F_{\varepsilon}=F_{t}^{\varepsilon, \zeta_{\alpha}(\varepsilon)}, G_{\varepsilon}=G_{t}^{\varepsilon, \zeta_{\alpha}(\varepsilon)}$. So we verify the hypothesis there.

Step 1. By (2.14) we know that the integration by parts formula (1.15) holds with $H_{\beta, \varepsilon}=K_{\beta}\left(F_{t}^{\varepsilon, \zeta_{\alpha}(\varepsilon)}, G_{t}^{\varepsilon, \zeta_{\alpha}(\varepsilon)}\right)$. By $(2.15)$ we obtain for every $\kappa \in\left(\frac{1}{\eta_{0}}, \lambda\right)$ (with $\zeta=\zeta_{\alpha}(\varepsilon)$ )

$$
\sup _{|\beta| \leq q}\left\|H_{\beta, \varepsilon}\right\|_{p} \leq \frac{C}{t^{\frac{2+\nu}{\nu}(12 q-4)}} e^{C \Gamma_{\varepsilon}^{\gamma}}\left(\varepsilon^{-q} \zeta^{-\nu q}+e^{-\Gamma_{\varepsilon}^{\kappa}} \zeta^{-2 \nu q}\right) .
$$

We use (2.3) and (2.4) in order to obtain

$$
e^{C \Gamma_{\varepsilon}^{\gamma}}\left(\varepsilon^{-q} \zeta^{-\nu q}+e^{-\Gamma_{\varepsilon}^{\kappa}} \zeta^{-2 \nu q}\right) \leq C \varepsilon^{-c}\left(\left(\varepsilon \zeta^{\nu}\right)^{-q}+\varepsilon^{A} \varepsilon^{-2 \nu q(1+\gamma+\alpha) /(1-\nu)}\right)
$$

for every $c>0$ and $A \geq 1$. Notice that $\varepsilon \zeta_{\alpha}^{\nu}(\varepsilon)=\varepsilon^{(1+\nu(q+\alpha)) /(1-\nu)}$. Taking $A \geq 2 \nu q(1+\gamma+\alpha) /(1-\nu)$ we obtain

$$
\sup _{|\beta| \leq q}\left\|H_{\beta, \varepsilon}\right\|_{p} \leq \frac{C}{t^{\frac{2+\nu}{\nu}(12 q-4)}} \times \varepsilon^{-q \times \frac{1+\nu(\gamma+\alpha)}{1-\nu}-c}
$$

and this means that (1.16) is verified with

$$
\widehat{H}_{q, p}=\frac{C}{t^{\frac{2+\nu}{\nu}(12 q-4)}}, \quad b=\frac{1+\nu(\gamma+\alpha)}{1-\nu}, \quad a=\frac{c}{b} .
$$

Let $\delta>0$. Taking $h$ sufficiently large we have $\frac{1}{2 h}(12(2 h+q+2)-4) \leq 12(1+\delta)$ so that

$$
\widehat{H}_{2 h+q+2, p}^{1 / 2 h} \leq \frac{C}{t^{\frac{2+\nu}{\nu} \times 12(1+\delta)}}
$$

Step 2. Let us verify (1.17). Using (2.12) and (2.11)

$$
\begin{aligned}
\left\|1-G_{t}^{\varepsilon, \zeta_{\alpha}(\varepsilon)}\right\|_{2} & \left.\leq P^{1 / 2} \sup _{s \leq t}\left|V_{s}^{\varepsilon, \zeta_{\alpha}(\varepsilon)}\right| \geq \Gamma_{\varepsilon}\right) \\
& \leq C e^{-\frac{1}{2} \Gamma_{\varepsilon}^{\lambda^{\prime}}}\left(E\left(\sup _{s \leq t} e^{\left|V_{s}^{\varepsilon, \zeta_{\alpha}(\varepsilon)}\right|^{\lambda^{\prime}}}\right)\right)^{1 / 2} \leq C e^{-\frac{1}{2} \Gamma_{\varepsilon}^{\lambda^{\prime}}} \leq C \varepsilon^{A} .
\end{aligned}
$$

The last inequality is true for any $A \geq 1$. It is a consequence of $\lambda^{\prime} \eta_{0}>1$ and of (2.4). Recall that $F_{t}^{\varepsilon, \zeta_{\alpha}(\varepsilon)}$ is defined in (2.13). We have

$$
E\left(\left|V_{t}^{\varepsilon, \zeta_{\alpha}(\varepsilon)}-F_{t}^{\varepsilon, \zeta_{\alpha}(\varepsilon)}\right|\right) \leq C \zeta_{\alpha^{\frac{4+\nu}{2}}}(\varepsilon)=C \varepsilon^{(1+\gamma+\alpha) \frac{4+\nu}{2(1-\nu)}} \leq C \varepsilon^{1+\gamma+\alpha}
$$

Then, as a consequence of (3.1), for every $c>0$ we obtain

$$
E\left(\left|V_{t}-F_{t}^{\varepsilon, \zeta_{\alpha}(\varepsilon)}\right|\right) \leq \frac{C}{t^{\kappa-1}} \varepsilon^{1+\gamma+\alpha-c} .
$$


We conclude that (1.17) holds with

$$
C_{*}=\frac{C}{t^{\kappa-1}}, \quad \beta=1+\gamma+\alpha-c .
$$

Step 3. Now (3.4) ensures that, for sufficiently small $c>0$,

$$
\begin{aligned}
\beta-b\left(1+q+\frac{2}{p_{*}}\right) & =1+\gamma+\alpha-c-\left(1+q+\frac{2}{p_{*}}\right) \frac{1+\nu(\gamma+\alpha)}{1-\nu} \\
& >1+\gamma+\alpha-(1+\varphi(\alpha)) \frac{1+\nu(\gamma+\alpha)}{1-\nu}=0
\end{aligned}
$$

the last equality being a consequence of (3.2) (this is the motivation of choosing $\varphi$ to be the solution of this equation).

So (1.20) holds (with $d=2$ ) and we are able to use Theorem 1.4. Notice that for every $\theta \geq 1$ and every $\lambda^{\prime}<\lambda^{\prime \prime}$ one may find $C$ such that $\Phi_{\lambda^{\prime}}^{\theta} \leq C \Phi_{\lambda^{\prime \prime}}$. So (2.11) gives $C_{\theta}(\Phi)<\infty$ (see (1.19) for the definition of $\left.C_{\theta}(\Phi)\right)$. By $(1.21)$

$$
\left\|\Phi_{\lambda^{\prime}} f_{t}\right\|_{q, p} \leq C \times\left(C_{*}+C_{\theta}(\Phi)\right) \times\left(h^{2 b} \widehat{H}_{2 h+q+d, p_{*}}^{1 / 2 h}\right)^{(1+\delta)\left(1+q+2 / p_{*}\right)} .
$$

We denote $C^{\prime}=C_{\theta}(\Phi) \times h^{2 b(1+\delta)\left(1+q+2 / p_{*}\right)}$. Then, using (3.10) and (3.9)

$$
\begin{aligned}
\left\|\Phi_{\lambda^{\prime}} f_{t}\right\|_{q, p} & \leq \frac{C}{t^{\kappa-1+\frac{2+\nu}{\nu} \times 12\left(1+q+2 / p_{*}\right)(1+\delta)^{2}}} \\
& \leq \frac{C}{t^{\kappa-1+\frac{2+\nu}{\nu} \times 12(1+\varphi(\alpha))(1+\delta)^{2}}} .
\end{aligned}
$$

We take $\delta>0$ sufficiently small so that $12(1+\delta)^{2} \leq 13$ and $\mathbf{A}$ is proved.

Proof of B. We use A with $q=0$. Let $0<\alpha^{\prime}<\varphi(\alpha) \wedge 2$. Since $\alpha^{\prime}<2$ we may find $p>1$ such that $\frac{2}{p_{*}}=\alpha^{\prime}<\varphi(\alpha)$, so (3.4) holds for this $p$. $\operatorname{By}(3.5)\left\|f_{t}\right\|_{p} \leq\left\|\Phi_{\lambda^{\prime}} f_{t}\right\|_{p} \leq C t^{-\kappa^{\prime}}$ with $\kappa^{\prime}$ given in (3.6). Using Hölder's inequality we get $\left(A_{\alpha^{\prime}, \kappa^{\prime}}\right)$ :

$$
f_{t}(\operatorname{Ball}(w, \varepsilon)) \leq\left\|f_{t}\right\|_{p} \times \varepsilon^{2 / p_{*}} \leq C t^{-\kappa^{\prime}} \times \varepsilon^{\alpha^{\prime}} .
$$

Proof of C. Take first $k=1$. We know that $\left(A_{0,0}\right)$ holds, and by hypothesis, $\varphi(0)>0$. Then, according to $\mathbf{B},\left(A_{\alpha^{\prime}, \kappa^{\prime}}\right)$ holds for every $\alpha^{\prime}<\varphi(0) \wedge 2=\alpha_{1} \wedge 2$ with $\kappa^{\prime}=0-1+\frac{13(2+\nu)}{\nu}(1+\varphi(0))=\kappa_{1}$. So our assertion holds for $k=1$.

Suppose now that the property is true for $k$ and let us check it for $k+1$. Suppose first that $\alpha_{k}>2$. Then $\alpha_{k+1}>\alpha_{k}>2$. By the recurrence hypothesis, for $\alpha<2=\alpha_{k} \wedge 2=\alpha_{k+1} \wedge 2$ the hypothesis $\left(A_{\alpha, \kappa_{k}}\right)$ holds. Since $\kappa_{k+1}>\kappa_{k}$, the hypothesis $\left(A_{\alpha, \kappa_{k+1}}\right)$ holds as well.

Suppose now that $\alpha_{k} \leq 2$ and take $\alpha^{\prime}<\alpha_{k+1} \wedge 2=\varphi\left(\alpha_{k}\right) \wedge 2$. Since $\varphi(\alpha) \uparrow \varphi\left(\alpha_{k}\right)$ as $\alpha \uparrow \alpha_{k}$, we may find $\alpha<\alpha_{k}=\alpha_{k} \wedge 2$ such that $\alpha^{\prime}<\varphi(\alpha) \wedge 2$. By the recurrence hypothesis we know that $\left(A_{\alpha, \kappa_{k}}\right)$ holds and then, using $\mathbf{B}$, we obtain $\left(A_{\alpha^{\prime}, \kappa_{k+1}}\right)$.

D. By (3.7) we may find $\alpha<\alpha_{k} \wedge 2$ such that $q+\frac{2}{p_{*}}<\varphi(\alpha)$. By $\mathbf{C}$ we know that $\left(A_{\alpha, \kappa_{k}}\right)$ holds. Then we may use $\mathbf{A}$ and (3.5) gives (3.8).

Proof of Theorem 1.1. We will work with the sequences $\alpha_{k}$ and $\kappa_{k}$ given in (3.3). Recall that $\alpha_{k} \uparrow \alpha_{*}$ with $\alpha_{*}=\varphi\left(\alpha_{*}\right)$. Direct computation give

$$
\alpha_{*}=\frac{-(\gamma+2)+\sqrt{(\gamma+2)^{2}+4\left(\frac{\gamma}{\nu}-2 \gamma-1\right)}}{2}
$$


and

$$
\begin{array}{lll}
\alpha_{*}>0 & \Leftrightarrow \quad \nu<\frac{\gamma}{2 \gamma+1} \\
\alpha_{*}>1 & \Leftrightarrow \quad \nu<\frac{\gamma}{3 \gamma+4} \\
\alpha_{*}>2 & \Leftrightarrow \quad \nu<\frac{\gamma}{4 \gamma+9} .
\end{array}
$$

If $\nu<\frac{\gamma}{2 \gamma+1}$ then $\varphi(0)>0$ so we may use the point $\mathbf{A}$ in Lemma 3.1 with $q=0$. We obtain $f_{t}(d v)=f_{t}(v) d v$ so the point $\mathbf{A}$ in Theorem 1.1 is proved.

Proof of B.b. If $\frac{\gamma}{4 \gamma+9}<\nu<\frac{\gamma}{2 \gamma+1}$ we have $\alpha_{*} \leq 2$ so that $\alpha_{k}<2$ for every $k \in N$. If $p<\frac{2}{2-\alpha_{*}}$ then $\frac{2}{p_{*}}<\alpha_{*}$ so we may find $k$ such that $\frac{2}{p_{*}}<\alpha_{k+1}<2$. Using the point $\mathbf{D}$ in Lemma 3.1 (with $q=0$ ) we obtain $\Phi_{\lambda^{\prime}} f_{t} \in L^{p}\left(R^{2}\right)$.

Proof of C.b. If $\frac{\gamma}{4 \gamma+9}<\nu<\frac{\gamma}{3 \gamma+4}$ we have $\alpha_{*} \in(1,2]$ so that $1<\frac{2}{3-\alpha_{*}}$. We take $1<p<\frac{2}{3-\alpha_{*}}$ and then $1+\frac{2}{p_{*}}<\alpha_{*}$. We take $k$ sufficiently large in order to have $1+\frac{2}{p_{*}}<\alpha_{k+1}$ and then, as above, by D in Lemma 3.1, we obtain $\Phi_{\lambda^{\prime}} f_{t} \in W^{1, p}\left(\mathbb{R}^{2}\right)$.

Proof of B a If $\nu<\frac{\gamma}{4 \gamma+9}$ then $\alpha_{*}>2$. Recall that $\alpha_{k} \uparrow \alpha_{*}$ and define $k_{*}=\min \left\{k: \alpha_{k} \geq 2\right\}$. By $\mathbf{C}$ in Lemma 3.1, for every $\alpha<2$ the property $\left(A_{\alpha, \kappa_{k_{*}}}\right)$ holds. We denote $\psi(\alpha)=\varphi(\alpha)-\alpha$. Notice that $\psi$ is decreasing on $(0,2)$. For $k<k_{*}$ we have $\alpha_{k}<2=\alpha_{k_{*}} \wedge 2$ so that $\psi(2)<\psi\left(\alpha_{k}\right)=\alpha_{k+1}-\alpha_{k}$. It follows that

$$
2>\alpha_{k_{*}-1}=\sum_{k=0}^{k_{*}-2}\left(\alpha_{k+1}-\alpha_{k}\right)>\left(k_{*}-1\right) \psi(2)
$$

which gives $k_{*}-1 \leq 2 / \psi(2)$ and so $k_{*}+1 \leq 2(\varphi(2)-1) /(\varphi(2)-2)$. Since $\alpha_{k} \leq \alpha_{*}=\varphi\left(\alpha_{*}\right)$ we have for every $k$ (see $(3.3))$

$$
\kappa_{k} \leq \kappa_{k-1}+\frac{13(2+\nu)}{\nu}\left(1+\varphi\left(\alpha_{*}\right)\right)-1 \leq \ldots \leq k\left(\frac{13(2+\nu)}{\nu}\left(1+\alpha_{*}\right)-1\right) .
$$

This yields

$$
\kappa_{k_{*}+1} \leq\left(k_{*}+1\right) \times\left(\frac{13(2+\nu)}{\nu}\left(1+\alpha_{*}\right)-1\right) \leq \frac{2(\varphi(2)-1)}{\varphi(2)-2}\left(\frac{13\left(1+\alpha_{*}\right)(2+\nu)}{\nu}-1\right)=\eta
$$

with $\eta$ given in (1.5).

We use now the point $\mathbf{D}$ in Lemma 3.1 with $q=0$. Recall that $\varphi(2)>2\left(\right.$ see (1.7)) Since $\varphi\left(\alpha_{k_{*}} \wedge 2\right)=$ $\varphi(2)>2>\frac{2}{p_{*}}$ for every $p>1$, we obtain $g_{t} \in \cap_{p>1} L^{p}\left(R^{2}\right)$. Moreover, taking $k=k_{*}$ in (3.8) we get

$$
\left\|g_{t}\right\|_{p} \leq \frac{C}{t^{\kappa_{k *+1}}} \leq \frac{C}{t^{\eta}}
$$

Proof of C.a. If $q \in\{1,2\}$, we need $\frac{2}{p_{*}}<\varphi(2)-q$. This gives $p<2 /(q+2-\varphi(2))=p_{q}$ with $p_{q}, q=1,2$ given in (1.9). And using (3.8) we obtain

$$
\left\|g_{t}\right\|_{q, p} \leq \frac{C}{t^{\kappa_{k_{*}+1}}} \leq \frac{C}{t^{\eta}} \quad \text { for } \quad p<p_{q}
$$

Proof of Corollary 1.2 Recall that $\nu<\frac{\gamma}{2 \gamma+1}$ is equivalent with $\varphi(0)>0$. So we may find $p>1$ such that $\frac{2}{p_{*}}<\varphi(0)$. Using $\mathbf{D}$ in Lemma 3.1 with $q=0$ and $k=0$ we obtain $\left\|\Phi_{\lambda^{\prime}} f_{t}\right\|_{p} \leq \frac{C}{t^{\kappa_{1}}}$ with $\kappa_{1}$ given 
in (3.3) (which coincides with $\kappa$ defined in (1.12)) Then

$$
\begin{aligned}
f_{t}\left(B_{R}^{c}(0)\right) & =\int 1_{B_{R}^{c}(0)}(v) \Phi_{\lambda^{\prime}}^{-1}(v) \Phi_{\lambda^{\prime}}(v) f_{t}(v) d v \\
& \leq\left(\int 1_{B_{R}^{c}(0)}(v) e^{-p_{*}|v|^{\lambda^{\prime}}} d v\right)^{1 / p_{*}}\left\|\Phi_{\lambda^{\prime}} f_{t}\right\|_{p} \\
& \leq e^{-\frac{1}{2} R^{\lambda^{\prime}}}\left(\int 1_{B_{R}^{c}(0)}(v) e^{-\frac{p_{*}}{2}|v|^{\lambda^{\prime}}} d v\right)^{1 / p_{*}} \frac{C}{t^{\kappa}} \\
& \leq \frac{C}{t^{\kappa}} e^{-\frac{1}{2} R^{\lambda^{\prime}}} .
\end{aligned}
$$

Proof of Theorem 1.3. Since $\nu<\frac{\gamma}{4 \gamma+9}$ we may use C.a in Theorem 1.1 with $q=1$ and we obtain $\left\|\Phi_{\lambda^{\prime}} f\right\|_{1, p} \leq C t^{-\eta}$ for every $p<p_{1}$ (with $p_{1}$ given in (1.9) and $\eta$ given in (1.5)). Notice also that we have $p_{1}>2$ so we may use the point $\mathbf{C}$ in Theorem 1.4: (1.23) gives (1.13). Notice also that if $\lambda^{\prime}<\lambda^{\prime \prime}$ then $\widehat{\nabla} \Phi_{\lambda^{\prime}}(x) \leq C \Phi_{\lambda^{\prime \prime}}(x)$. So (1.25) gives (1.14).

\section{Appendix: A regularity criterion based on interpolation}

Let us first recall some results obtained in [2] concerning the regularity of a measure $\mu$ on $\mathbb{R}^{d}$. Fix $k, q, h \in \mathbb{N}$, with $h \geq 1$, and $p>1$ (we denote by $p_{*}$ the conjugate of $p$ ). For $f \in C^{\infty}\left(R^{d}\right.$ ) we define

$$
\begin{aligned}
\|f\|_{k, \infty} & =\sum_{0 \leq|\alpha| \leq k} \sup _{x \in R^{d}}\left|\partial^{\alpha} f(x)\right| \\
\|f\|_{k, h, p} & =\sum_{0 \leq|\alpha| \leq k}\left(E\left(\int_{R^{d}}(1+|x|)^{h}\left|\partial^{\alpha} f(x)\right|^{p} d x\right)\right)^{1 / p} \\
\|f\|_{k, p} & =\|f\|_{k, 0, p}=\sum_{0 \leq|\alpha| \leq k}\left\|\partial^{\alpha} f\right\|_{p} .
\end{aligned}
$$

Here $\alpha=\left(\alpha_{1}, \ldots, \alpha_{m}\right) \in\{1, \ldots, d\}^{m}$, is a multi-index of length $|\alpha|=m$ and $\partial^{\alpha}$ is the derivative associated to $\alpha$. Moreover for two measures $\mu, \nu$ we consider the distance

$$
d_{k}(\mu, \nu)=\sup \left\{\left|\int f d \mu-\int f d \nu\right|:\|f\|_{k, \infty} \leq 1\right\} .
$$

For $k=0$ this is the total variation distance and for $k=1$ this is the Fortèt Mourier distance.

For a finite measure $\mu$ and for a sequence of absolutely continuous finite measures $\mu_{n}(d x)=f_{n}(x) d x$ with $f_{n} \in C^{2 h+q}\left(\mathbb{R}^{d}\right)$, we define

$$
\pi_{k, q, h, p}\left(\mu,\left(\mu_{n}\right)_{n}\right)=\sum_{n=0}^{\infty} 2^{n\left(k+q+d / p_{*}\right)} d_{k}\left(\mu, \mu_{n}\right)+\sum_{n=0}^{\infty} \frac{1}{2^{2 n h}}\left\|f_{n}\right\|_{2 h+q, 2 h, p}
$$

and

$$
\bar{\pi}_{k, q, h, p}(\mu)=\inf \left\{\pi_{k, q, h, p}\left(\mu,\left(\mu_{n}\right)_{n}\right): \mu_{n}(d x)=f_{n}(x) d x, \quad f_{n} \in C^{2 h+q}\left(\mathbb{R}^{d}\right)\right\} .
$$

Remark 4.1 Notice that $\pi_{k, q, h, p}$ is a particular case of $\pi_{k, q, h, \mathbf{e}}$ treated in [2]: just choose the Young function $\mathbf{e}(x) \equiv \mathbf{e}_{p}(x)=|x|^{p}$ (see Example 1 in [2]). Moreover, $\pi_{k, q, h, p}$ is strongly related to interpolation spaces. More precisely, $\bar{\pi}_{k, q, h, p}$ is equivalent with the interpolation norm of order $\rho=\frac{k+q+d / p_{*}}{2 h}$ between the spaces $W_{*}^{k, \infty}$ (the dual of $W^{k, \infty}$ ) and $W^{2 h+q, 2 h, p}=\left\{f:\|f\|_{2 h+q, 2 h, p}<\infty\right\}$ (see [6] for example). This is proved in [2], see Section 2.4 and Appendix B. So the inequality (4.6) below implies that the Sobolev space $W^{q, p}$ is included in the above interpolation space. However we prefer to stick to an elementary framework and to derive directly the consequences of (4.6) - see Lemma 4.3 and Lemma 4.2 below. 
The following result is the key point in our approach:

Lemma 4.2 Let $p>1, k, q \in \mathbb{N}$ and $h \in \mathbb{N}_{*}=\mathbb{N} /\{0\}$ be given. There exists a constant $C_{*}$ (depending on $k, q, h$ and $p$ only) such that the following holds. Let $\mu$ be a finite measure for which $\bar{\pi}_{k, q, h, p}(\mu)$ is finite. Then $\mu(d x)=f(x) d x$ with $f \in W^{q, p}$ and

$$
\|f\|_{q, p} \leq C_{*} \times \bar{\pi}_{k, q, h, p}(\mu) .
$$

This is Proposition 2.5 in [2] in the particular case $e(x)=e_{p}(x)=|x|^{p}$. See also Proposition 3.2.1 in $[3]$. So we will not give here the proof. We will use the following consequence:

Lemma 4.3 Let $p>1, k, q \in \mathbb{N}$ and $h \in \mathbb{N}_{*}$ be given and set

$$
\rho_{h}(q):=\frac{k+q+d / p_{*}}{2 h} .
$$

We consider an increasing sequence $\theta(n) \geq 1, n \in \mathbb{N}$ such that $\lim _{n} \theta(n)=\infty$ and $\theta(n+1) \leq \Theta \times \theta(n)$ for some constant $\Theta \geq 1$. Moreover, we consider a sequence of measures $\mu_{n}(d x)=f_{n}(x) d x$ with $f_{n} \in C^{2 h+q}\left(\mathbb{R}^{d}\right), n \in \mathbb{N}$ such that

$$
\left\|f_{n}\right\|_{2 h+q, 2 h, p} \leq \theta(n) .
$$

Let $\mu$ be a finite measure such that, for some $\eta>0$,

$$
\limsup _{n} d_{k}\left(\mu, \mu_{n}\right) \times \theta^{\rho_{h}(q)+\eta}(n)<\infty .
$$

Then $\mu(d x)=f(x) d x$ with $f \in W^{q, p}$.

Moreover, fix $n_{*} \in \mathbb{N}, \delta>0$ and $\eta>0$ such that (4.9) holds. We set

$$
\begin{aligned}
A(\delta) & =|\mu|\left(\mathbb{R}^{d}\right) \times 2^{l(\delta)(1+\delta)\left(q+k+d / p_{*}\right)} \quad \text { with } \\
l(\delta) & =\min \left\{l \geq 1: 2^{l^{\prime} \times \frac{\delta}{1+\delta}} \geq l^{\prime}, \forall l^{\prime} \geq l\right\} \\
B(\eta) & =\sum_{l=1}^{\infty} \frac{l^{2\left(q+k+d / p_{*}+\eta\right)}}{2^{2 h \eta l}}, \\
C_{h, n_{*}}(\eta) & =\sup _{n \geq n_{*}} d_{k}\left(\mu, \mu_{n}\right) \times \theta^{\rho_{h}(q)+\eta}(n) .
\end{aligned}
$$

Then

$$
\|f\|_{q, p} \leq C_{*}\left(\Theta+A(\delta) \theta\left(n_{*}\right)^{\rho_{h}(q)(1+\delta)}+B(\eta) C_{h, n_{*}}(\eta)\right)
$$

with $C_{*}$ the constant in (4.6) and $\rho_{h}(q)$ given in (4.7).

Proof of Lemma 4.3. We will produce a sequence of measures $\nu_{l}(d x)=g_{l}(x) d x, l \in \mathbb{N}$ such that

$$
\pi_{k, q, h, p}\left(\mu,\left(\nu_{l}\right)_{l}\right) \leq \Theta+A(\delta) \theta\left(n_{*}\right)^{\rho_{h}(q)(1+\delta)}+B(\eta) C_{h, n_{*}}(\eta)<\infty .
$$

Then by Lemma 4.2 one gets $\mu(d x)=f(x) d x$ with $f \in W^{q, p}$, and (4.14) follows from (4.6)). Let us stress that the $\nu_{l}$ 's will be given by a suitable subsequence $\mu_{n(l)}, l \in \mathbb{N}$.

Step 1. We define

$$
n(l)=\min \left\{n: \theta(n) \geq \frac{2^{2 h l}}{l^{2}}\right\}
$$

and we notice that

$$
\frac{1}{\Theta} \theta(n(l)) \leq \theta(n(l)-1)<\frac{2^{2 h l}}{l^{2}} \leq \theta(n(l))
$$


Moreover we recall that $n_{*}$ is given and we define

$$
l_{*}=\min \left\{l: \frac{2^{2 h l}}{l^{2}} \geq \theta\left(n_{*}\right)\right\} .
$$

Since

$$
\theta\left(n\left(l_{*}\right)\right) \geq \frac{2^{2 h l_{*}}}{l_{*}^{2}} \geq \theta\left(n_{*}\right)
$$

it follows that $n\left(l_{*}\right) \geq n_{*}$.

We take now $\varepsilon(\delta)=\frac{h \delta}{1+\delta}$ which gives $\frac{2 h}{2(h-\varepsilon(\delta))}=1+\delta$. And we take $l(\delta) \geq 1$ such that $2^{l \delta /(1+\delta)} \geq l$ for $l \geq l(\delta)$ (see (4.11)). Since $h \geq 1$ it follows that $\varepsilon(\delta) \geq \frac{\delta}{1+\delta}$ so that, for $l \geq l(\delta)$ we also have $2^{l \varepsilon(\delta)} \geq l$. Now we check that

$$
2^{2(h-\varepsilon(\delta)) l_{*}} \leq 2^{2 h l(\delta)} \theta\left(n_{*}\right) .
$$

If $l_{*} \leq l(\delta)$ then the inequality is evident (recall that $\theta(n) \geq 1$ for every $n$ ). And if $l_{*}>l(\delta)$ then $2^{l_{*} \varepsilon(\delta)} \geq l_{*}$. By the very definition of $l_{*}$ we have

$$
\frac{2^{2 h\left(l_{*}-1\right)}}{\left(l_{*}-1\right)^{2}}<\theta\left(n_{*}\right)
$$

so that

$$
2^{2 h l_{*}} \leq 2^{2 h}\left(l_{*}-1\right)^{2} \theta\left(n_{*}\right) \leq 2^{2 h} \times 2^{2 l_{*} \varepsilon(\delta)} \theta\left(n_{*}\right)
$$

and, since $l(\delta) \geq 1$, this gives (4.16).

Step 2. We define

$$
\begin{aligned}
\nu_{l} & =0 \quad \text { if } \quad l<l_{*} \\
& =\mu_{n(l)} \quad \text { if } \quad l \geq l_{*}
\end{aligned}
$$

and we estimate $\pi_{k, q, h, p}\left(\mu,\left(\nu_{l}\right)_{l}\right)$. First, by (4.8) and (4.15)

$$
\sum_{l=l_{*}}^{\infty} \frac{1}{2^{2 h l}}\left\|f_{n(l)}\right\|_{q+2 h, 2 h, p} \leq \sum_{l=l_{*}}^{\infty} \frac{1}{2^{2 h l}} \theta(n(l)) \leq \Theta \sum_{l=l_{*}}^{\infty} \frac{1}{l^{2}} \leq \Theta .
$$

Then we write

$$
\sum_{l=1}^{\infty} 2^{\left(q+k+d / p_{*}\right) l} d_{k}\left(\mu, \nu_{l}\right)=S_{1}+S_{2}
$$

with

$$
S_{1}=\sum_{l=1}^{l_{*}-1} 2^{\left(q+k+d / p_{*}\right) l} d_{k}(\mu, 0), \quad S_{2}=\sum_{l=l_{*}}^{\infty} 2^{\left(q+k+d / p_{*}\right) l} d_{k}\left(\mu, \mu_{n(l)}\right) .
$$

Since $d_{k}(\mu, 0) \leq d_{0}(\mu, 0) \leq|\mu|\left(\mathbb{R}^{d}\right)$ we use (4.16) and we obtain

$$
\begin{aligned}
S_{1} & \leq|\mu|\left(\mathbb{R}^{d}\right) \times 2^{\left(q+k+d / p_{*}\right) l_{*}}=|\mu|\left(\mathbb{R}^{d}\right) \times\left(2^{2(h-\varepsilon(\delta)) l_{*}}\right)^{\left(q+k+d / p_{*}\right) / 2(h-\varepsilon(\delta))} \\
& \leq|\mu|\left(\mathbb{R}^{d}\right) \times\left(2^{2 h l(\delta)} \theta\left(n_{*}\right)\right)^{\rho_{h}(q)(1+\delta)}=A(\delta) \theta\left(n_{*}\right)^{\rho_{h}(q)(1+\delta)} .
\end{aligned}
$$

If $l \geq l_{*}$ then $n(l) \geq n\left(l_{*}\right) \geq n_{*}$ so that, using (4.13) first and (4.15) then, we obtain

$$
d_{k}\left(\mu, \mu_{n(l)}\right) \leq \frac{C_{h, n_{*}}(\eta)}{\theta^{\rho_{h}(q)+\eta}(n(l))} \leq C_{h, n_{*}}(\eta)\left(\frac{l^{2}}{2^{2 h l}}\right)^{\rho_{h}(q)+\eta}=\frac{C_{h, n_{*}}(\eta)}{2^{\left(q+k+d / p_{*}\right) l}} \times \frac{l^{2\left(\rho_{h}(q)+\eta\right)}}{2^{2 h \eta l}} .
$$

We conclude that

$$
S_{2} \leq C_{h, n_{*}}(\eta) \sum_{l=l_{*}}^{\infty} \frac{l^{2\left(\rho_{h}(q)+\eta\right)}}{2^{2 \eta h l}} \leq C_{h, n_{*}}(\eta) \times B(\eta)
$$

We give now a consequence of the above result which is more readable. 
Proposition 4.4 Let $q, k, d \in \mathbb{N}$ and $p>1$ be fixed. We consider a family of measures $\mu_{\varepsilon}(d x)=$ $f_{\varepsilon}(x) d x, \varepsilon>0$ with $f_{\varepsilon} \in C^{\infty}\left(\mathbb{R}^{d}\right)$ and a finite measure $\mu$ on $\mathbb{R}^{d}$ which verify the following hypothesis. There exists $\varepsilon_{*}>0, \beta>0, a \geq 0, b \geq 0, C_{0} \geq 1$ and $Q_{h}(q, p) \geq 1$ such that for every $h \in \mathbb{N}_{*}$ and every $0<\varepsilon<\varepsilon_{*}$

$$
\begin{aligned}
\text { i) } d_{k}\left(\mu_{\varepsilon}, \mu\right) & \leq C_{0} \varepsilon^{\beta}, \\
\text { ii) }\left\|f_{\varepsilon}\right\|_{2 h+q, 2 h, p} & \leq Q_{h}(q, p) \varepsilon^{-b(2 h+q+a)}, \\
\text { iii }) \quad r & :=\beta-b\left(k+q+d / p_{*}\right)>0 .
\end{aligned}
$$

We denote

$$
h_{*}=\frac{1}{\varepsilon_{*}} \vee \frac{b(q+a)\left(k+q+d / p_{*}\right)}{r} \vee \frac{q+a}{2} .
$$

Then, $\mu(d x)=f(x) d x$ with $f \in W^{q, p}\left(\mathbb{R}^{d}\right)$. Moreover, for every $\delta>0$, there exists a constant $C \geq 1$, depending on $q, k, d, p, \delta, \beta, r$ and $a, b$ only (but which does not depend neither on $h$ nor on $C_{0}$ ), such that for every $h \geq h_{*}$ one has

$$
\|f\|_{q, p} \leq C \times C_{0} \times\left(h^{2 b} Q_{h}^{1 / 2 h}(q, p)\right)^{(1+\delta)\left(k+q+d / p_{*}\right)}
$$

Proof. All over this proof $C$ designs a constant which depends on $q, k, d, p, \delta, \beta, r$ and $a, b$ only (we stress that in particular it may depend on $C_{*}$ from (4.6)). We will use Lemma 4.3. We take

$$
\eta=\frac{r}{2 b(2 h+q+a)} \wedge\left(\delta \rho_{h}(q)\right)
$$

with $\rho_{h}(q)$ given in (4.7). For $h \geq h_{*}$ one has $\rho_{h}(q) b(q+a) \leq \frac{r}{2}$, and, by definition, $r=\beta-2 h \rho_{h}(q) b$. Using also (4.22) we obtain

$$
\begin{aligned}
\beta-\left(\rho_{h}(q)+\eta\right) b(2 h+q+a) & =\left(\beta-2 h \rho_{h}(q) b\right)-\rho_{h}(q) b(q+a)-\eta b(2 h+q+a) \\
& \geq r-\frac{r}{2}-\frac{r}{2}=0 .
\end{aligned}
$$

It follows that for every $\varepsilon \leq \varepsilon_{*}$ we have

$$
d_{k}\left(\mu_{\varepsilon}, \mu\right)\left\|f_{\varepsilon}\right\|_{2 h+q, 2 h, p}^{\rho_{h}(q)+\eta} \leq C_{0} Q_{h}^{\rho_{h}(q)+\eta}(q, p) \varepsilon^{\beta-\left(\rho_{h}(q)+\eta\right) b(2 h+q+a)} \leq C_{0} Q_{h}^{\rho_{h}(q)(1+\delta)}(q, p) .
$$

We take now $\varepsilon_{n}=\frac{1}{n}$ and $n_{*}=h$ and we define

$$
\begin{aligned}
g_{n} & =0 \quad \text { if } \quad n<n_{*} \\
& =f_{\varepsilon_{n}} \quad \text { if } \quad n \geq n_{*} .
\end{aligned}
$$

We will use Lemma 4.3 for $\nu_{n}(d x)=g_{n}(x) d x$ so we have to identify the quantities defined there. We define

$$
\begin{aligned}
& \theta(n)=Q_{h}(q, p) n^{b(2 h+q+a) \quad \text { if } \quad n \geq n_{*}} \\
& \theta(n)=\theta\left(n_{*}\right) \quad \text { if } \quad n \leq n_{*} .
\end{aligned}
$$

By (4.18) we have $\left\|g_{n}\right\|_{2 h+q, 2 h, p} \leq \theta(n)$ and moreover, for $n \geq n_{*}=h$, we have

$$
\frac{\theta(n+1)}{\theta(n)}=\left(1+\frac{1}{n}\right)^{n \times \frac{b(2 h+q+a)}{n}} \leq e^{2 b+\frac{b(q+a)}{h}} \leq e^{3 b} .
$$


We conclude that $\Theta \leq e^{3 b}$. We estimate now $B(\eta)$ defined in (4.12). Noticed first that

$$
\begin{aligned}
\frac{1}{\eta h} & =\frac{2 b(2 h+q+a)}{r h} \vee \frac{2}{\delta\left(q+k+d / p_{*}\right)} \\
& \leq \frac{6 b}{r} \vee \frac{2}{\delta\left(q+k+d / p_{*}\right)}=: C_{1}
\end{aligned}
$$

so $\eta h \geq 1 / C_{1}$. Then

$$
B(\eta)=\sum_{l=1}^{\infty} \frac{l^{2\left(q+k+d / p_{*}+\eta\right)}}{2^{2 h \eta l}} \leq \sum_{l=1}^{\infty} \frac{l^{2\left(q+k+d / p_{*}+\eta\right)}}{2^{2 l / C_{1}}} \leq C .
$$

Moreover, since $h \geq \frac{1}{2}(q+a)$ it follows that

$$
\rho_{h}(q)(1+\delta) b(2 h+q+a) \leq 2(1+\delta) b\left(k+q+d / p_{*}\right)
$$

and consequently (recall that $n_{*}=h$ )

$$
\begin{aligned}
\theta\left(n_{*}\right)^{\rho_{h}(q)(1+\delta)} & =Q_{h}^{\rho_{h}(q)(1+\delta)}(q, p) n_{*}^{\rho_{h}(q)(1+\delta) b(2 h+q+a)} \\
& \leq Q_{h}^{\rho_{h}(q)(1+\delta)}(q, p) h^{2(1+\delta) b\left(k+q+d / p_{*}\right)}
\end{aligned}
$$

Finally we notice that, by (4.23), the constant $C_{h, n_{*}}(\eta)$ defined in (4.13) verifies

$$
C_{h, n_{*}}(\eta) \leq C_{0} Q_{h}^{\rho_{h}(q)(1+\delta)}(q, p) .
$$

As for $A(\delta)$ defined in (4.10), this is already a constant $C$ (which does not depend on $h$ and on $C_{0}$ ). Now we use (4.14) and we obtain

$$
\|f\|_{q, p} \leq C\left(1+Q_{h}^{\rho_{h}(q)(1+\delta)}(q, p) h^{2 b(1+\delta)\left(k+q+d / p_{*}\right)}+C_{0} Q_{h}^{\rho_{h}(q)(1+\delta)}(q, p)\right)
$$

which gives (4.21).

\subsection{Proof of Theorem 1.4}

The aim of this section is to prove Theorem 1.4 so we consider the framework given there: we have a family of random variables $F_{\varepsilon}$ and $G_{\varepsilon}, \varepsilon>0$ such that the integration by parts formula (1.15) and the estimate (1.16) hold; we also have a random variable $F$ such that the estimate (1.17) holds. We define the measures $\mu$ and $\mu_{\varepsilon}$ by

$$
\int \phi d \mu=E(\phi(F)) \quad \text { and } \quad \int \phi d \mu_{\varepsilon}=E\left(\phi\left(F_{\varepsilon}\right) G_{\varepsilon}\right) .
$$

As a consequence of (1.15) and of (1.16) we have $\mu_{\varepsilon}(d x)=f_{\varepsilon}(x) d x$ with $f_{\varepsilon} \in C^{\infty}\left(\mathbb{R}^{d}\right)$.

We also consider a function $\Phi \in \mathcal{C}$ (so in particular $\Phi$ verifies (1.18)). All these hypothesis are in force in this section.

Moreover, for $v \in \mathbb{R}^{d}$ we construct the "exterior rectangle" $A_{v}$ in the following way. For $y \in \mathbb{R}$ we denote $I_{y}=(y, \infty)$ if $y \geq 0$ and $I_{y}=(-\infty, y)$ if $y<0$. And for $v=\left(v_{1}, \ldots, v_{d}\right)$ we define

$$
A_{v}=\prod_{i=1}^{d} I_{v_{i}}
$$

We will first prove the following two Lemmas. 
Lemma 4.5 For every $q, h \in \mathbb{N}$ and $p>1$ there exists some constants $C$ and $\theta$ (depending on $q, h, d$ and $p$ only) and $\varepsilon_{*}>0$ such that, for every $\varepsilon \in\left(0, \varepsilon_{*}\right)$

$$
\left\|\Phi f_{\varepsilon}\right\|_{q, h, p} \leq C \times C_{\theta}(\Phi) \times \widehat{H}_{q+d, p_{*}} \times \varepsilon^{-b(q+d+a)}
$$

with $C_{\theta}(\Phi)$ given in (1.19) and $\widehat{H}_{q+d, p_{*}}$ given in (1.16).

Proof. We denote

$$
I_{q, h, p}(\Phi)(x)=\sup _{|\beta| \leq q} \int_{R^{d}}(1+|v|)^{h}\left|\partial^{\beta} \Phi(v)\right|^{p} 1_{A_{v}}(x) d v .
$$

By (1.15) (we use a formal computation which may be done rigorous by regularization by convolution)

$$
\begin{aligned}
\partial^{\alpha}\left(\Phi f_{\varepsilon}\right)(v) & =\sum_{(\beta, \gamma)=\alpha} \partial^{\beta} \Phi(v) \partial^{\gamma} f_{\varepsilon}(v)=\sum_{(\beta, \gamma)=\alpha} \partial^{\beta} \Phi(v) E\left(\partial^{\gamma} \delta_{0}\left(F_{\varepsilon}-v\right) G_{\varepsilon}\right) \\
& =\sum_{(\beta, \gamma)=\alpha} \partial^{\beta} \Phi(v) E\left(1_{A_{v}}\left(F_{\varepsilon}\right) H_{(\gamma, 1, \ldots, d), \varepsilon}\right) .
\end{aligned}
$$

Using Hölder's inequality first and then (1.16)

$$
\begin{aligned}
\left|\partial^{\alpha}\left(\Phi f_{\varepsilon}\right)(v)\right| & \leq \sum_{(\beta, \gamma)=\alpha}\left|\partial^{\beta} \Phi(v)\right| P^{1 / p}\left(F_{\varepsilon} \in A_{v}\right)\left\|H_{(\gamma, 1, \ldots, d), \varepsilon}\right\|_{p_{*}} \\
& \leq \sum_{|\beta| \leq q}\left|\partial^{\beta} \Phi(v)\right| P^{1 / p}\left(F_{\varepsilon} \in A_{v}\right) \times \widehat{H}_{q+d, p_{*}} \varepsilon^{-b(q+d+a)} .
\end{aligned}
$$

This gives

$$
\begin{aligned}
\left\|\Phi f_{\varepsilon}\right\|_{q, h, p} & =\sum_{|\alpha| \leq q}\left(\int(1+|v|)^{h}\left|\partial^{\alpha}\left(\Phi f_{\varepsilon}\right)(v)\right|^{p} d v\right)^{1 / p} \\
& \leq C \widehat{H}_{q+d, p_{*}} \varepsilon^{-b(q+d+a)} \sum_{|\beta| \leq q}\left[\int(1+|v|)^{h}\left|\partial^{\beta} \Phi(v)\right|^{p} E\left(1_{F_{\varepsilon} \in A_{v}}\right) d v\right]^{1 / p} \\
& =C \widehat{H}_{q+d, p_{*}} \varepsilon^{-b(q+d+a)}\left[E\left(I_{q, h, p}(\Phi)\left(F_{\varepsilon}\right)\right)\right]^{1 / p} .
\end{aligned}
$$

By using (1.18) we may find some constants $c_{1}$ and $c_{2}$ such that for every $\gamma$ with $|\gamma| \leq q$

$$
\left|\partial^{\gamma} \Phi(v)\right|^{p} \leq \frac{c_{1}}{(1+|v|)^{h+d+1}}|\Phi(v)|^{c_{2} p}
$$

so we get

$$
E\left(I_{q, h, p}(\Phi)\left(F_{\varepsilon}\right)\right) \leq c_{1} E\left(\int_{R^{d}}(1+|v|)^{-(d+1)}|\Phi(v)|^{c_{2} p} 1_{A_{v}}\left(F_{\varepsilon}\right) d v\right) .
$$

If $x \in A_{v}$ then $|v| \leq|x|$ and since $\Phi \in \mathcal{C}$ this implies $\Phi(v) \leq C \Phi(x)$. So the above term is upper bounded by

$$
C E\left(\int_{R^{d}}(1+|v|)^{-(d+1)}\left|\Phi\left(F_{\varepsilon}\right)\right|^{c_{2} p} 1_{A_{v}}\left(F_{\varepsilon}\right) d v\right) \leq C E\left(\left|\Phi\left(F_{\varepsilon}\right)\right|^{c_{2} p}\right) \leq C \times C_{\theta}(\Phi)
$$

with $\theta=c_{2} p$.

Lemma 4.6 We recall that by (1.17)

$$
\left\|1-G_{\varepsilon}\right\|_{2}+\left\|F-F_{\varepsilon}\right\|_{1} \leq C_{*} \varepsilon^{\beta} .
$$

Then, for every $\delta>0$ there exists $\theta(\delta) \geq 1$ and $C \geq 1$ such that

$$
\left.d_{1}\left(\Phi \mu_{\varepsilon}, \Phi \mu\right) \leq C\left(C_{*}+C_{\theta(\delta)}(\Phi)\right)\right) \varepsilon^{\beta(1-\delta)}
$$

with $C_{\theta}(\Phi)$ defined in (1.19). 
Proof. Let $\phi$ with $\|\phi\|_{1, \infty} \leq 1$. We estimate first

$$
\left|E\left((\phi \Phi)\left(F_{\varepsilon}\right)\left(1-G_{\varepsilon}\right)\right)\right| \leq\|\phi\|_{\infty}\left\|\Phi\left(F_{\varepsilon}\right)\right\|_{2}\left\|1-G_{\varepsilon}\right\|_{2} \leq C_{2}(\Phi) \times C_{*} \varepsilon^{\beta} .
$$

Then we write

$$
\left|E\left((\phi \Phi)\left(F_{\varepsilon}\right)-(\phi \Phi)(F)\right)\right| \leq E \int_{0}^{1}\left|\nabla(\phi \Phi)\left(\lambda F+(1-\lambda) F_{\varepsilon}\right)\left(F-F_{\varepsilon}\right)\right| d \lambda .
$$

Using (1.18) and the fact that $\Phi$ is a convex function

$$
\begin{aligned}
\left|\nabla(\phi \Phi)\left(\lambda F+(1-\lambda) F_{\varepsilon}\right)\right| & \leq c_{1}\|\phi\|_{1, \infty}\left|\Phi\left(\lambda F+(1-\lambda) F_{\varepsilon}\right)\right|^{c_{2}} \\
& \leq C\left(\lambda|\Phi(F)|^{c_{2}}+(1-\lambda)\left|\Phi\left(F_{\varepsilon}\right)\right|^{c_{2}}\right) .
\end{aligned}
$$

It follows that the last term in (4.30) is upper bounded by

$$
C\left(E\left(|\Phi(F)|^{c_{1}}\left|F-F_{\varepsilon}\right|\right)+E\left(\left|\Phi\left(F_{\varepsilon}\right)\right|^{c_{1}}\left|F-F_{\varepsilon}\right|\right)\right) .
$$

We take $K>0$ and we write

$$
E\left(\left|\Phi\left(F_{\varepsilon}\right)\right|^{c_{1}}\left|F-F_{\varepsilon}\right|\right)=I_{K}\left(F_{\varepsilon}\right)+J_{K}\left(F_{\varepsilon}\right)
$$

with

$$
I_{K}\left(F_{\varepsilon}\right)=E\left(\left|\Phi\left(F_{\varepsilon}\right)\right|^{c_{1}}\left|F-F_{\varepsilon}\right| 1_{\left\{\left|\Phi\left(F_{\varepsilon}\right)\right|^{c_{1}} \leq K\right\}}\right) \leq K C_{*} \varepsilon^{\beta}
$$

and

$$
\begin{aligned}
J_{K}\left(F_{\varepsilon}\right) & =E\left(\left|\Phi\left(F_{\varepsilon}\right)\right|^{c_{1}}\left|F-F_{\varepsilon}\right| 1_{\left\{\left|\Phi\left(F_{\varepsilon}\right)\right|^{c_{1}}>K\right\}}\right) \\
& \leq\left(\|F\|_{2}+\left\|F_{\varepsilon}\right\|_{2}\right)\left(E\left(\left|\Phi\left(F_{\varepsilon}\right)\right|^{2 c_{1}} 1_{\left\{\left|\Phi\left(F_{\varepsilon}\right)\right|^{c_{1}}>K\right\}}\right)\right)^{1 / 2} \\
& \leq \frac{\left(\|F\|_{2}+\left\|F_{\varepsilon}\right\|_{2}\right)}{K^{\theta}}\left(E\left(\left|\Phi\left(F_{\varepsilon}\right)\right|^{2 c_{1}(1+\theta)}\right)^{1 / 2} .\right.
\end{aligned}
$$

By $(1.18)\|F\|_{2}+\left\|F_{\varepsilon}\right\|_{2} \leq C \times C_{c_{2}}^{1 / 2}(\Phi)$ so finally

$$
J_{K}\left(F_{\varepsilon}\right) \leq \frac{C}{K^{\theta}} C_{c_{2} \vee 2 c_{1}(1+\theta)}(\Phi) .
$$

These estimates hold for every $\theta \geq 1$ and $K>0$. In order to optimize we take $K=\varepsilon^{-\beta /(1+\theta)}$ and we obtain

$$
I_{K}\left(F_{\varepsilon}\right)+J_{K}\left(F_{\varepsilon}\right) \leq C \times C_{c_{2} \vee 2 c_{1}(1+\theta)}(\Phi) \times \varepsilon^{\beta \times \frac{\theta}{1+\theta}} .
$$

A similar inequality holds with $F$ instead of $F_{\varepsilon}$, so, using (4.29) as well we obtain

$$
\left|E\left((\phi \Phi)\left(F_{\varepsilon}\right)-(\phi \Phi)(F)\right)\right| \leq C\left(C_{*}+C_{c_{2} \vee 2 c_{1}(1+\theta)}(\Phi)\right) \times \varepsilon^{\beta \times \frac{\theta}{1+\theta}}
$$

Then, taking $\theta=(1-\delta) / \delta$ we get $(4.28)$.

We are now ready to give:

Proof of Theorem 1.4. We will use the Proposition 4.4 with $k=1$ for the measures $(\Phi \mu)(d x)$ and $\left(\Phi \mu_{\varepsilon}\right)(d x)=\Phi(x) f_{\varepsilon}(x) d x$ with $\mu$ and $\mu_{\varepsilon}$ given in (4.24). By (1.20) we may find (and fix) $\delta>0$ such that

$$
b-\delta>b\left(1+q+\frac{d}{p_{*}}\right)
$$

By (4.28) we have

$$
\left.d_{1}\left(\Phi \mu_{\varepsilon}, \Phi \mu\right) \leq C\left(C_{0}+C_{\theta(\delta)}(\Phi)\right)\right) \varepsilon^{\beta(1-\delta)}
$$


so (4.17) holds with the constant $\left.C_{0}=C\left(C_{*}+C_{\theta(\delta)}(\Phi)\right)\right)$. By (4.26)

$$
\left\|\Phi f_{\varepsilon}\right\|_{2 h+q, 2 h, p} \leq C \times C_{\theta}(\Phi) \times \widehat{H}_{2 h+q+d, p_{*}} \varepsilon^{-b(2 h+q+d+a)} .
$$

So (4.18) holds with $Q_{h}(q, p)=C C_{\theta}(\Phi) \widehat{H}_{2 h+q+d, p_{*}}$. Notice that $a$ from (4.18) is replaced by $a^{\prime}=a+d$. This changes nothing except the value of $h_{*}$ (see (4.20)) which anyway, is not explicit in our statement. As a consequence of (4.31) hypothesis (4.19) is verified so we are able to use Proposition 4.4 in order to obtain $(\Phi \mu)(d x)=g(x) d x$ with $g \in W^{q, p}\left(\mathbb{R}^{d}\right)$. It follows that $\mu(d x)=f(x) d x$ with $f=g / \Phi$ and by (4.21) (the value of $\theta$ changes from a line to another and we use the inequality $C_{\theta}^{\theta^{\prime}}(\Phi) \leq C_{\theta \times \theta^{\prime}}(\Phi)$ )

$$
\begin{aligned}
\|\Phi f\|_{q, p} & \leq C \times\left(C_{*}+C_{\theta}(\Phi)\right) \times\left(h^{2 b} Q_{h}^{1 / 2 h}(q, p)\right)^{(1+\delta)\left(k+q+d / p_{*}\right)} \\
& \leq C \times\left(C_{*}+C_{\theta}(\Phi)\right) \times\left(h^{2 b} C_{\theta}^{1 / 2 h}(\Phi) \widehat{H}_{2 h+q+d, p_{*}}^{1 / 2 h}\right)^{(1+\delta)\left(k+q+d / p_{*}\right)} \\
& \leq \Gamma_{\Phi, \theta}(q, h, p) .
\end{aligned}
$$

So (1.21) is proved.

We prove now the point $\mathbf{C}$. If the above inequality holds with $q=1$ and $p>d$, then, by Morrey's lemma $\Phi f$ is $\chi$ - Hölder continuous with $\chi=1-\frac{d}{p}$ and

$$
\|\Phi f\|_{\infty} \leq\|\Phi f\|_{C^{0, \chi}} \leq C\|\Phi f\|_{1, p} \leq C \Gamma_{\Phi}(1, h, p) .
$$

So we obtain (1.23). Let us prove (1.25). Recall the definition of $\widehat{\nabla} \Phi(x)$ defined in (1.24). We write

$$
(\Phi f)(y)-(\Phi f)(x)=\Phi(x)(f(y)-f(x))+(\Phi(y)-\Phi(x)) f(x)
$$

so that, if $|x-y| \leq 1$, then

$$
\begin{aligned}
|\Phi(x)(f(y)-f(x))| & \leq\|\Phi f\|_{C^{0, \chi}}|x-y|^{\chi}+\widehat{\nabla} \Phi(x)|f(x)||x-y| \\
& \leq C\|\Phi f\|_{1, p}|x-y|^{\chi}+\frac{\widehat{\nabla} \Phi(x)}{\Phi(x)}\|\Phi f\|_{1, p}|x-y| \\
& \leq C \Gamma_{\Phi}(1, h, p)|x-y|^{\chi}+\widehat{\nabla} \Phi(x) \times \frac{\Gamma_{\Phi}(1, h, p)}{\Phi(x)}|x-y|
\end{aligned}
$$

where, in order to obtain the second inequality we have used (1.23). So (1.25) is proved.

\section{Appendix 2: The integration by parts formula.}

The aim of this section is to give a hint to the proof of (2.14) and of the estimates (2.15) and (2.16). The integration by parts formula (2.14) has been established in [5] by using a version of Malliavin calculus for jump processes introduced in [4]. All this machinery is quit heavy and we are not able to give here a detailed technical view (we refer to [5] for a complete presentation). We just try to give an overlook which permits to the reader to understand which are the main objects and arguments involved in the proof of these results. And also to precise the dependence with respect to $t$ of the constants in (2.15) and (2.16). 


\subsection{Real chock and fictive chock representation}

A first step is to give some appropriate alternative representations for $V_{t}^{\varepsilon, \zeta}$, solution of the equation (see $(2.7))$ :

$$
V_{t}^{\varepsilon, \zeta}=V_{0}+\int_{0}^{t} \int_{E \times \mathbb{R}_{+}} A(\theta)\left(V_{s-}^{\varepsilon, \zeta}-v\right) 1_{\left\{u \leq \varphi_{\varepsilon}^{\gamma}\left(\left|V_{s-}^{\varepsilon, \zeta}-v\right|\right)\right\}} I_{\zeta}(\theta) N(d s, d \theta, d v, d u) .
$$

We recall $E=\left[-\frac{\pi}{2}, \frac{\pi}{2}\right] \times \mathbb{R}^{2}$ and $N(d t, d \theta, d v, d u)$ is a Poisson point measure on $E \times \mathbb{R}_{+}$with intensity measure $d t \times b(\theta) d \theta \times d f_{t}(d v) \times d u$ where $f_{t}(d v)$ is the solution (which exists and is unique) of the equation (1.1).

Step 1. In a first stage we use some change of variable in order to write the above equation in an alternative form which is appropriate for our calculus (see Section 3 in [5] for details; the motivation of this new representation is just technical). Using the Skorohod representation theorem we may find a measurable function $v_{t}:[0,1] \rightarrow \mathbb{R}^{2}$ such that for every $\psi: \mathbb{R}^{2} \rightarrow \mathbb{R}_{+}$

$$
\int_{0}^{1} \psi\left(v_{t}(\rho)\right) d \rho=\int_{\mathbb{R}^{2}} \psi(v) f_{t}(d v)
$$

This allows to replace the measure $f_{t}(d v)$ on $\mathbb{R}^{2}$ by $d \rho$ on $[0,1]$.

Moreover, for $x \in\left(0, \frac{\pi}{2}\right]$, let $G(x)=\int_{x}^{\pi / 2} b(\theta) d \theta$ and let $g:(0, \infty) \rightarrow\left(0, \frac{\pi}{2}\right]$ be the inverse of $G$, that is $G(g(z))=z$ (since $b(\theta) \simeq|\theta|^{-(1+\nu)}$ by assumption, it follows that $G(x) \simeq \nu^{-1}\left(x^{-\nu}-(\pi / 2)^{-\nu}\right)$ and $\left.g(z) \simeq(1+z)^{-\nu}\right)$. For $z<0$ we define $g(z)=-g(-z)$. With this construction we will have

$$
\int_{-\pi / 2}^{\pi / 2} \psi(\theta) b(\theta) d \theta=\int_{\mathbb{R}_{*}}^{\infty} \psi(g(z)) d z
$$

and this allows to replace the measure $b(\theta) d \theta$ on $\left(-\frac{\pi}{2}, \frac{\pi}{2}\right)$ by $d z$ on $\mathbb{R}_{*}:=\mathbb{R} \backslash\{0\}$.

Finally we consider a function $\mathbf{I}_{\zeta}: \mathbb{R} \rightarrow[0,1]$ which is smooth, with all derivatives bounded and such that $\mathbf{I}_{\zeta}(z)=1$ for $|z| \leq G(\zeta)$ and $\mathbf{I}_{\zeta}(z)=0$ for $|z| \geq G(\zeta)+1$. And we choose the function $I_{\zeta}$ in equation (5.1) in such a way that

$$
\mathbf{I}_{\zeta}(z)=I_{\zeta}(g(z))
$$

Then we may write the equation (5.1) as

$$
V_{t}^{\varepsilon, \zeta}=V_{0}+\int_{0}^{t} \int_{0}^{1} \int_{-G(\zeta)-1}^{G(\zeta)+1} \int_{0}^{2 \Gamma_{\varepsilon}^{\gamma}} A(g(z))\left(V_{s-}^{\varepsilon, \zeta}-v_{s}(\rho)\right) 1_{\left\{u \leq \varphi_{\varepsilon}^{\gamma}\left(\left|V_{s-}^{\varepsilon, \zeta}-v_{s}(\rho)\right|\right)\right\}} \mathbf{I}_{\zeta}(z) M(d s, d \rho, d z, d u)
$$

with $M$ a Poisson point measure on $[0, T] \times[0,1] \times \mathbb{R}_{*} \times(0, \infty)$ with intensity measure $m(d s, d \rho, d z, d u)=$ $d s d \rho d z d u$. Notice that we may take $u \leq 2 \Gamma_{\varepsilon}^{\gamma}$ because we know that $\varphi_{\varepsilon}(v) \leq \Gamma_{\varepsilon}$ (we use $2 \Gamma_{\varepsilon}$ instead of $\Gamma_{\varepsilon}$ just for technical reasons).

Step 2. Since $m$ is a finite measure we may represent the above equation by using a compound Poisson process as follows. We consider a standard Poisson process $J_{t}^{\varepsilon}=\sum_{k=1}^{\infty} 1_{\left\{T_{k} \leq t\right\}}$ of parameter $\lambda=4(G(\zeta)+1) \Gamma_{\varepsilon}^{\gamma}$ and a sequence $\left(\bar{R}_{k}, \bar{Z}_{k}, \bar{U}_{k}\right), k \in \mathbb{N}$ of independent random variables, uniformly distributed on $[0,1] \times[-G(\zeta)-1, G(\zeta)+1] \times\left[0,2 \Gamma_{\varepsilon}^{\gamma}\right]$, which are independent of $J$ (all these objects depend on $\varepsilon$ and $\zeta$, but, as they are fixed, we do not mention it in the notation). Now the equation (5.3) may be written as

$$
V_{t}^{\varepsilon, \zeta}=V_{0}+\sum_{T_{k} \leq t} A\left(g\left(\bar{Z}_{k}\right)\right)\left(V_{T_{k-1}, \zeta}^{\varepsilon, \zeta}-v_{T_{k}}\left(\bar{R}_{k}\right)\right) 1_{\left\{\bar{U}_{k} \leq \varphi_{\varepsilon}^{\gamma}\left(\left|V_{T_{k-1}, \zeta}^{\varepsilon, v_{T_{k}}}\left(\bar{R}_{k}\right)\right|\right)\right\}} \mathbf{I}_{\zeta}\left(\bar{Z}_{k}\right)
$$

This equation is known as "the fictive chock" representation (see for example [25]). 
Step 3. The idea of the approach by means of the Malliavin calculus is to look to $V_{t}^{\varepsilon, \zeta}$ as a functional $f\left(\bar{Z}_{1}, \ldots, \bar{Z}_{J_{t}}\right)$ of $\bar{Z}_{k}, k=1, \ldots, J_{t}^{\varepsilon}$ and to use an elementary integration by parts formula based on the (uniform) law of $\bar{Z}_{k}$. But this is not possible directly because of the indicator function of the set $\left\{\bar{U}_{k} \leq\right.$ $\left.\varphi_{\varepsilon}^{\gamma}\left(\left|V_{T_{k-1}}^{\varepsilon, \zeta}-v_{T_{k}}\left(\bar{R}_{k}\right)\right|\right)\right\}$ which appears in the equation : as a consequence $\bar{Z}_{k} \rightarrow f\left(\bar{Z}_{1}, \ldots, \bar{Z}_{J_{t}^{\varepsilon}}\right)=V_{T_{k}}^{\varepsilon, \zeta}$ is not differentiable. In order to avoid this difficulty we introduce the so called "real chock representation" that we present now. We consider the equation

$$
V_{t}^{\varepsilon, \zeta}=V_{0}+\sum_{T_{k} \leq t} A\left(g\left(Z_{k}\right)\right)\left(V_{T_{k-1}, \zeta}^{\varepsilon}-v_{T_{k}}\left(R_{k}\right)\right) \mathbf{I}_{\zeta}\left(Z_{k}\right)
$$

This is the same equation as (5.4) but the indicator function disappears. But now the law of $\left(R_{k}, Z_{k}\right)$ is no more the uniform law (as above). We define this law as follows: we assume that conditionally to $T_{k}=t$ and $V_{T_{k}}^{\varepsilon, \zeta}=w$ the law of $\left(R_{k}, Z_{k}\right)$ is given by

$$
P_{t, w}\left(R_{k} \in d \rho, Z_{k} \in d z\right)=q_{t, w}(\rho, z) d z d \rho
$$

with

$$
q_{t, w}(\rho, z)=\frac{1}{\lambda} \varphi_{\varepsilon}^{\gamma}(|w-v(\rho)|) 1_{\{|z| \leq G(\zeta)+1\}}+g_{t, w}(z) .
$$

Here $g_{t, w}(z)$ is an auxiliary smooth function which is null on $\{|z| \leq G(\zeta)+1\}$ and which is chosen in such a way that $\iint q_{t, w}(\rho, z) d \rho d z=1$ (it plays the role of a cemetery and does not come on in the computations). Notice that $q_{t, w}(\rho, z) d z d \rho$ gives the precise way in which the law of $\left(R_{k}, Z_{k}\right)$ (and so the law of the jump) depends on the position $V_{T_{k}-}^{\varepsilon, \zeta}=w$ of the particle. One may check (see Section 3 in [5] for details) that the law of the solution of the equation (5.5) coincides with the law of $V_{t}^{\varepsilon, \zeta}$, the solution of (5.4). So we may (and do) work with $V_{t}^{\varepsilon, \zeta}$ solution of (5.5) now on. This is the "real chock representation". Now the machinery which produces $V_{t}^{\varepsilon, \zeta}$ as a function of $Z_{k}$ is a smooth function and we may use a differential calculus for it. Notice that we know nothing about the regularity of the function $\rho \rightarrow v_{t}(\rho)$ and consequently we are not able to use $R_{k}$ - we will just use $Z_{k}$. We also mention that $V_{T_{k}}^{\varepsilon, \zeta}$ is a function of $T_{i}, R_{i}, Z_{i}, i=1, \ldots, k$ so we will use the (slightly abusive notation)

$$
V_{T_{k}}^{\varepsilon, \zeta}=\mathcal{H}_{k}\left(\omega, Z_{1}, \ldots, Z_{k}\right)
$$

where $\omega$ indicates the dependence on $T_{i}, R_{i}, i=1, \ldots, k$.

\subsection{Finite dimensional Malliavin calculus.}

In this section we present the results concerning the Malliavin calculus based on the random variables $Z_{k}, k \in \mathbb{N}$ from the previous section. We add two standard normal random variables $Z_{-1}, Z_{0}$ which are independent of $Z_{k}, k=1,2, \ldots$ as well (they correspond to the two dimensional standard normal random variable $Z$ in introduced (2.13)). Given $t>0$ we denote $Z_{t}=\left(Z_{-1}, Z_{0}, Z_{1}, \ldots, Z_{J_{t}^{\varepsilon}}\right)$. The law of $Z_{t}$ is absolutely continuous with respect to the Lebesgue measure on $\mathbb{R}^{J_{t}^{\varepsilon}+2}$ and has the density

$$
p_{t}(\omega, z)=c_{t} e^{\frac{\left|z_{-1}\right|^{2}+\left|z_{0}\right|^{2}}{2}} \prod_{k=1}^{J_{t}^{\varepsilon}} q_{T_{k}, \mathcal{H}_{k-1}\left(\omega, z_{1}, \ldots, z_{k-1}\right)}\left(R_{k}, z_{k}\right) .
$$

The integration by parts formula which we derive in the sequel will be based on the logarithmic derivative of this density. In order to avoid border terms in the integration by parts formula we introduce the weights

$$
\pi_{-1}=\pi_{0}=1 \quad \text { and } \quad \pi_{k}=a_{\zeta}\left(Z_{k}\right)
$$

where $a_{\zeta}$ is a smooth version of $1_{(1, G(\zeta))}(z)$. 
We follow the strategy established in Malliavin calculus. A simple functional $F$ is a random variable of the form $F=h\left(\omega, Z_{t}\right)$ where $\omega$ designees the dependence on $T_{i}, R_{i}, i \in \mathbb{N}$ and $z \rightarrow h(\omega, z)$ is a smooth function on $\mathbb{R}_{t}^{J_{t}^{\varepsilon}+2}$ which has bounded derivatives of any order. Then we define the derivatives

$$
D_{k} F=\pi_{k} \partial_{z_{k}} h\left(\omega, Z_{t}\right)
$$

and for a multi-index $\kappa=\left(k_{1}, \ldots, k_{m}\right) \in\left\{-1,0,1, \ldots, J_{t}^{\varepsilon}\right\}^{m}$ we denote $|\kappa|=m$ and we define

$$
D^{\kappa} F=D_{k_{m}} \ldots D_{k_{1}} F \text {. }
$$

For a $d$ dimensional simple functional $F=\left(F_{1}, \ldots, F_{d}\right)$ the Malliavin covariance matrix is given by

$$
\sigma_{F}^{i, j}=\left\langle D F_{i}, D F_{j}\right\rangle=\sum_{k=-1}^{J_{t}^{\varepsilon}} D_{k} F_{i} \times D_{k} F_{j}
$$

and, for a one dimensional simple functional $F$, the divergence operator is defined by

$$
L F=-\sum_{k=-1}^{J_{t}^{\varepsilon}}\left[\frac{1}{\pi_{k}} D_{k}\left(\pi_{k} D_{k} F\right)+D_{k} F \times D_{k} \log p_{t}\left(\omega, Z_{t}\right)\right] .
$$

Using elementary integration by parts on $\mathbb{R}^{J_{t}^{\varepsilon}+2}$ one obtains the following duality formula

$$
E(\langle D F, D G\rangle)=E(F L G)=E(G L F) .
$$

We will work with the norms

$$
|F|_{m}=|F|+\sum_{1 \leq|\kappa| \leq m}\left|D^{\kappa} F\right|
$$

The standard arguments from Malliavin calculus give the following integration by parts formula (see Theorem 1 and 3 in [4] )): let $G$ and $F=\left(F_{1}, \ldots, F_{d}\right)$ be simple functionals. We suppose that $1 / \operatorname{det} \sigma_{F} \in$ $\cap_{p>1} L^{p}$. Then for every $\psi \in C_{b}^{\infty}\left(R^{d}\right)$ and every multi-index $\beta=\left(\beta_{1}, \ldots, \beta_{q}\right) \in\{1, \ldots, d\}^{q}$ one has

$$
\left.E\left(\partial^{\beta} \psi(F) G\right)\right)=E\left(\psi(F) K_{\beta}(F, G)\right)
$$

where $K_{\beta}(F, G)$ is a random variable which verifies

$$
\begin{aligned}
\left|K_{\beta}(F, G)\right| & \leq C \times \bar{K}_{\beta}(F, G) \times\left|\operatorname{det} \sigma_{F}\right|^{-(3 q-1)} \text { with } \\
\bar{K}_{\beta}(F, G) & =|G|_{q}\left(1+|F|_{q+1}\right)^{q(6 d+1)}\left(1+\sum_{j=1}^{q} \sum_{k_{1}+\ldots+k_{j}=q-j} \prod_{i=1}^{j}|L F|_{k_{i}}\right) .
\end{aligned}
$$

In our approach (see Section 3) we choose $\zeta(\varepsilon)=\varepsilon^{(1+\gamma+\alpha) /(1-\nu)}$ and we use above estimate for (see (2.13)):

$$
F_{t}^{\varepsilon, \zeta(\varepsilon)}=\sqrt{u_{\zeta(\varepsilon)}(t)} Z+V_{t}^{\varepsilon, \zeta(\varepsilon)} \quad \text { with } \quad u_{\zeta}(t)=t \zeta^{4+\nu} .
$$

We also recall that in $(2.12)$ we have introduced $G_{t}^{\varepsilon, \zeta}$ which is a smooth version of the indicator function of the set $\left\{\sup _{s \leq t}\left|V_{s}^{\zeta, \varepsilon}\right| \leq \Gamma_{\varepsilon}\right\}$. In particular, for every $q$, we have $\left|G_{t}^{\varepsilon, \zeta}\right|_{q}=0$ on $\left\{\sup _{s \leq t}\left|V_{s}^{\zeta, \varepsilon}\right|>\Gamma_{\varepsilon}\right\}$. It follows that

$$
\bar{K}_{\beta}\left(F_{t}^{\varepsilon, \zeta(\varepsilon)}, G_{t}^{\varepsilon, \zeta(\varepsilon)}\right)=\bar{K}_{\beta}\left(F_{t}^{\varepsilon, \zeta(\varepsilon)}, G_{t}^{\varepsilon, \zeta(\varepsilon)}\right) 1_{\left\{\sup _{s \leq t}\left|V_{s}^{\zeta, \varepsilon}\right| \leq \Gamma_{\varepsilon}\right\}} .
$$


Remark 5.1 The main difficulty in our approach comes from the estimate of $L F_{t}^{\varepsilon, \zeta(\varepsilon)}$ which blows up as $\varepsilon \rightarrow 0$. In order to understand this we stress that the definition of $L F_{t}^{\varepsilon, \zeta(\varepsilon)}$ involves

$$
\partial_{z_{k}} \log p_{\varepsilon, t}(\omega, z)=\sum_{i=k}^{J_{t}^{\varepsilon}} \partial_{z_{r}} \log q_{T_{i}, \mathcal{H}_{i-1}\left(\omega, z_{1}, \ldots, z_{i-1}\right)}\left(R_{i}, z_{i}\right) .
$$

If $q_{t, w}(\rho, z)$ does not depend on $w$ (this means that the law of the jump does not depend on the position of the particle) then only the first term corresponding to $i=k$ in the sum is non null. But if it does depend (and this is our case), then all the terms are non null because of $\mathcal{H}_{i-1}\left(\omega, z_{1}, \ldots, z_{i-1}\right)$ depends on $z_{k}$ for every $i=k+1, \ldots, J_{i}^{\varepsilon}$ (the perturbation of $z_{k}$ propagates in the future). So we have $J_{t}^{\varepsilon}$ terms in the sum and, since $E\left(J_{t}^{\varepsilon}\right) \rightarrow \infty$ as $\varepsilon \rightarrow 0$, this generates a blow-up and we have to give an accurate estimate of it. It represents the main difficulty in our approach.

Our aim now is to give an upper bound for the $L^{p}$ norm of $K_{\beta}\left(F_{t}^{\varepsilon, \zeta}, G_{t}^{\varepsilon, \zeta}\right)$ more precisely (see (2.15))

$$
\left\|K_{\beta}\left(F_{t}^{\varepsilon, \zeta}, G_{t}^{\varepsilon, \zeta}\right)\right\|_{p} \leq \frac{C e^{C \Gamma_{\varepsilon}^{\gamma}}}{t^{4(3 q-1) \times \frac{2+\nu}{\nu}}}\left(\varepsilon^{-q} \zeta^{-\nu q}+e^{-\Gamma_{\varepsilon}^{\kappa} \zeta^{-2 \nu q}}\right)
$$

with $q=|\beta|$.

Sketch of the proof. In Proposition 4.11 in [5] one proves that for each $p>1, l \in \mathbb{N}$ and $T>0$

$$
\begin{gathered}
E\left(1_{\left\{\sup _{[0, T]}\left|V_{s}^{\varepsilon, \zeta}\right| \leq \Gamma_{\varepsilon}\right\}} \sup _{[0, T]}\left|V_{s}^{\varepsilon, \zeta}\right|_{l}^{p}\right) \leq C e^{C \Gamma_{\varepsilon}^{\gamma} \quad \text { and }} \\
E\left(1_{\left\{\sup _{[0, T]}\left|V_{s}^{\varepsilon, \zeta}\right| \leq \Gamma_{\varepsilon}\right\}} \sup _{[0, T]}\left|L V_{s}^{\varepsilon, \zeta}\right|_{l}^{p}\right) \leq \frac{C e^{C \Gamma_{\varepsilon}^{\gamma}}}{\varepsilon^{p(l+1) \zeta^{\nu p}}}
\end{gathered}
$$

where $C$ is a constant which depends on $p, l$ and $T$. Using the above estimates (recall (5.12)) and Hölder's inequality one obtains

$$
\sup _{t \leq T}\left\|\bar{K}_{\beta}\left(F_{t}^{\varepsilon, \zeta}, G_{t}^{\varepsilon, \zeta}\right)\right\|_{p} \leq C e^{C \Gamma_{\varepsilon}^{\gamma}}\left(\varepsilon^{-q} \zeta^{-\nu q}+e^{-\Gamma_{\varepsilon}^{\kappa}} \zeta^{-2 \nu q}\right) .
$$

See the proof of Theorem 4.1 in [5] for detailed computations.

Moreover, in Proposition 4.4 in [5] one denotes $d_{t}=\operatorname{det} \sigma_{F_{t}^{\varepsilon, \zeta}}$ and proves that

$$
E\left(d_{t}^{-p}\right) \leq C_{p, t} e^{c_{p} \Gamma_{\varepsilon}^{\gamma}}
$$

Here $c_{p}$ is a constant which depends on $p$ and $C_{p, t}$ depends on $p$ but also on $t$. The dependence in $t$ is not specified there, so we will check here that

$$
C_{p, t}=c t^{-4 p \times \frac{2+\nu}{\nu}}
$$

We go in the proof of Proposition 4.4 in [5] and we find the inequality

$$
E\left(d_{t}^{-p}\right) \leq C_{p} e^{C_{p} \Gamma_{\varepsilon}^{\gamma}}\left(\int_{\xi \in R^{2}}|\xi|^{8 p-2} \exp \left(-c t|\xi|^{\nu /(2+\nu)}\right) d \xi\right)^{1 / 2} .
$$

Using the change of variable $\bar{\xi}=t^{(2+\nu) / \nu} \xi$ one obtains (5.15) so that

$$
\left\|d_{t}^{-1}\right\|_{p} \leq \frac{1}{t^{\frac{4(2+\nu)}{\nu}}} C_{p} e^{C_{p} \Gamma_{\varepsilon}^{\gamma}}
$$

This, together with (5.14) and Schwarz's inequality gives (5.13). 


\section{References}

[1] R. Alexandre, L. Desvillettes,C. Villani,B. Wennberg (2000). Entropy dissipation and long-range interactions, Arch.Rat.Mech.Anal. 152, 327-355.

[2] V. Bally, L. Caramellino (2017). Convergence and regularity of probability laws by using an interpolation method. Ann.. Probab. 45 1110-1159 Preprint arXiv:1409.3118.

[3] V. Bally, L. Caramellino, R. Cont (2016): Stochastic integration by parts and functional Itô calculus. Advanced Courses in Mathematics - CRM Barcelona, Birkhäuser.

[4] V. Bally, E. Clement (2011). Integration by parts formulas and applications to equations with jumps. Probab. Theory Related Fields 151, 613-657.

[5] V. Bally, N. Fournier (2011). Regularization properties of the 2D homogeneous Boltzmann equation without cutoff. Probab. Theory Related Fields 151, 659-704.

[6] C. Bennett, R. Sharpley (1988). Interpolation of operators. Academic Press INC.

[7] K. Bichteler, J.B. Gravereau J. Jacod (1987). Malliavin calculus for processes with jumps. Gordon and Breach science publishers, New York.

[8] J.M. Bismut (1983). Calcul des variations stochastique et processus de sauts. Z. Wahrsch. Verw. Gebiete 63, 147-235.

[9] N. Bouleau, L. Denis(2015). Dirichlet forms and methods for Poisson point measures and Lévy processes. Probability Theory and Stochastic Modelling, 76, Springer.

[10] A. Debussche, N. Fournier (2013). Existence of densities for stable-like driven SDE's with Holder continuous coefficients. J. Funct. Anal. 264, 1757-1778.

[11] A. Debussche, M. Romito (2014). Existence of densities for the 3D Navier-Stokes equations driven by Gaussian noise. Probab. Theory Related Fields 158, 575-596.

[12] S. De Marco (2011). Smoothness and Asymptotic Estimates of densities for SDEs with locally smooth coefficients and Applications to square-root diffusions. Ann. Appl. Probab. 21, 1282-1321.

[13] N. Fournier (2000). Existence and regularity study for $2 D$ Bolzmann equation without cutoff by a probabilistic approach,. Ann. Appl. Probab. 10, 434-462.

[14] N. Fournier (2002). Jumping SDE's: absolute continuity using monotonicity. Stochastic Process. Appl. 98, 317-330.

[15] N. Fournier (2008). Smoothness of the law of some one-dimensional jumping SDE's with non constant rate of jump. Electron. J. Probab. 13, 135-156.

[16] N. Fournier (2015). Finiteness of entropy for the homogeneous Boltzmann equation with measure initial condition. Ann. Appl. Probab. 25, 860-897.

[17] N. Fournier, C. Mounot (2009). On the well-posenss of the spatially homogenous Bolzmann equation with a moderate angular singularity Comm. Math. Phys. 289, 803-824.

[18] N. Fournier, J. Printems (2010). Absolute continuity of some one-dimensional processes. Bernoulli 16, 343-360.

[19] I.M. Gamba, V. Panferov, C. Villani (2009). Upper Maxwellian bounds for the spacialy homogenuous Bolzmann equation Arch. Ration. Mech. Anal. 194, 253-282. 
[20] C. Graham,S Meleard (1999). Existance and regularity of a solution of a Kac equation without cutoff using the stochastic calculus of variations. Comm. Math. Phys.. 205, no 3, 551-569.

[21] H. Guerin,S Meleard,E. Nualart (2006). Exponential estimates for spatially homogeneous Landau equations via the Malliavin calculus. Journal of Func. Analysis, no. 2, 649-677.

[22] Y. Ishikawa (2013). Stochastic Calculus of variation for Jump Processes. De Gruyter Studies in Math. 54.

[23] V.N. Kolokoltsov (2006). On the regularity of the solutions of trhe space homogenuous Bolzmann equation with polynomial growing collision kernel. Advanced Studies in Contemporany Math. , no. 21, 9-38.

[24] R. Leandre (1985). Régularuté des processus de sauts gégénérés. Ann. Inst. H. Poincaré Probab. Statist. 21, 125-146.

[25] E. Pardoux,R Sentis,B. Lapeyre. (2003) Introduction to Monte-Carlo Methods for Transport and Diffusion Equations Oxford texts in applied and engineering mathematics, 6.

[26] H. TAnaka (1978). Probabilistic treatment of the Bolzmaznn equation of Maxwellian molecules Z, Wahrsch. und Verw. Gebiete 46, 67-105. 